\title{
Outer membrane protein folding and topology from a computational transfer free energy scale
}

\author{
Meishan Lin, Dennis Gessmann, Hammad Naveed, and Jie Liang* \\ University of Illinois at Chicago, Department of Bioengineering, Chicago, IL, 60607, USA \\ E-mail: jliang@uic.edu \\ Phone: +1 (312)3551789
}

\section{Supporting information}

\section{Reduced discrete state model of conformational space}

The reduce discrete state space $\Omega$ for the transmembrane (TM) region of an outer membrane $\beta$-barrel protein (OMP) is defined following reference. ${ }^{1}$ Briefly, each TM strand has a length $L+1=16$. Each residue on a TM strand interacts with neighboring strands through strong H-bond, non H-bond, or weak H-bond..$^{2-4}$ One strand $i$ can slide $d_{i} \in\{-l, \cdots, 0, \cdots, l\}$ residues away from its center position. A specific conformation of the TM region of an OMP with $N$ TM strands is defined by a vector $\mathbf{d}$ :

$$
\mathbf{d}=\left(d_{1}, \cdots, d_{N}\right)
$$

in which $d_{i}$ denotes the offset position of strand $i$. The full configuration space $\Omega$ is:

$$
\Omega=\left\{\mathbf{d} \mid \mathbf{d}=\left(d_{1}, \cdots, d_{N}\right) \in \mathbb{Z}^{N}\right\} .
$$

${ }^{*}$ To whom correspondence should be addressed 
Because each offset position $d_{i}$ can have a value from $-l$ to $l$, the size of the configuration space $\Omega$ is $(2 l+1)^{N}$. We used $l=3$ in this study.

\section{TM strands, side chain orientations, and center positions}

Sequences of TM strands are extracted from PDB structures of OMPs. The side-chain orientations of TM residues are determined by visualization using pymol. ${ }^{5}$ The inter-strand interaction type between two residues on neighboring strands, i.e., strong $\mathrm{H}$-bond, non $\mathrm{H}-$ bond and weak H-bond, is determined using the DSSP software. ${ }^{6}$ The midplanes of the lipid bilayers of OMPs are taken from the OPM server. ${ }^{7}$ The center residue of a TM strand is defined as the residue with the shortest distance to the mid-plane and is assigned the position index $i=0$. Position indice for other residues in the TM strands are assigned according to their relative positions to the center residue.

\section{Energy calculation of an OMP in a specific configuration}

The empirical potential function for calculating the energy of a TM strand is based on our previous empirical MsIP function, ${ }^{4}$ which was derived from structures of OMPs using the combinatorial permutation model as the reference state to resolve the coupling effects in short TM strands. For a specific configuration $d$, the empirical energy function consists of four terms. First, each residue contributes to the single-residue burial energy $E_{\mathrm{B}}(\mathbf{d})$ based on the region in which the residue is located (i.e., hydrophobic core, headgroup, and cap region) and its side-chain orientation (see reference ${ }^{4}$ for numerical details). Detailed analysis on the distribution of amino acid residues in different regions of membrane bilayers was described in our previous study. ${ }^{4}$ Second, each residue in the TM region interacts with residues in the neighboring strands through strong $\mathrm{H}$-bond interaction $\left(E_{\mathrm{SH}}\right)$ and non $\mathrm{H}$ bond interaction $\left(E_{\mathrm{NH}}\right)$ (see reference ${ }^{4}$ for numerical details). The summation of $E_{\mathrm{SH}}$ and $E_{\mathrm{NH}}$ is the inter-strand interaction energy $E_{\text {Inter }}(\mathbf{d})$. Third, each residue in the TM region of a strand interacts with two other residues on the neighboring strand through weak $\mathrm{H}-$ 
bond $\left(E_{\mathrm{WH}}(\mathbf{d})\right)$. Fourth, each residue interacts with the nearest residues on the same TM strand, which are of the same side-chain orientation. This term is the intra-strand interaction energy $E_{\text {Intra }}(\mathbf{d})$ (see reference ${ }^{8}$ for numerical details) . Following Wouters and Curmi, ${ }^{2}$ the strand-strand interaction network is that of the ideal anti-parallel $\beta$-sheet (Fig S1). Residues that do not form $\beta$-pairing interactions in the native structures usually have less favorable interactions in the canonical model and contribute little in the Boltzmann factor.

The strand energy $E(i ; \mathbf{d})$ for a TM strand $i$ in configuration $\mathbf{d}$ is then calculated as:

$$
\begin{aligned}
E(i ; \mathbf{d})= & w_{\mathrm{B}} \cdot E_{\mathrm{B}}(i ; \mathbf{d})+w_{\text {Intra }} \cdot E_{\text {Intra }}(i ; \mathbf{d})+w_{\text {Inter }} \cdot E_{\text {Inter }}(i ; \mathbf{d})+w_{\mathrm{WH}} \cdot E_{\mathrm{WH}}(i ; \mathbf{d}) \\
= & w_{\mathrm{B}} \cdot \sum_{k_{i}} E_{\mathrm{B}}\left(k_{i} ; d_{i}\right)+w_{\text {Intra }} \cdot \sum_{k_{i}} E_{\text {Intra }}\left(k_{i} ; d_{i}\right) \\
& +w_{\text {Inter }} \cdot\left[\sum_{k_{i}} \sum_{k_{i-1}}\left(E_{\text {Inter }}\left(k_{i}, k_{i-1} ; d_{i}, d_{i-1}\right)\right)+\sum_{k_{i}} \sum_{k_{i+1}}\left(E_{\text {Inter }}\left(k_{i}, k_{i+1} ; d_{i}, d_{i+1}\right)\right)\right] \\
& +w_{\mathrm{WH}} \cdot\left[\sum _ { k _ { i } } \sum _ { k _ { i - 1 } } \left(E_{\mathrm{WH}}\left(k_{i}, k_{i-1} ; d_{i}, d_{i-1}\right)+\sum_{k_{i}} \sum_{k_{i+1}}\left(E_{\mathrm{WH}}\left(k_{i}, k_{i+1} ; d_{i}, d_{i+1}\right)\right],(1)\right.\right.
\end{aligned}
$$

where $k_{i}$ is the position of a residue in strand $i, w_{\mathrm{B}}, w_{\text {Inter }}, w_{\mathrm{WH}}$, and $w_{\text {Intra }}$ are weights for the energy terms. We assigned weight values of 1.0, 0.6, 0.4, and 0.8 for $w_{\mathrm{B}}, w_{\text {Inter }}, w_{\mathrm{WH}}$, and $w_{\text {Intra }}$, respectively. Our results suggest that position-dependent residue burial term plays the most important role. As long as the rank order of $w_{\mathrm{B}} \geq w_{\text {Intra }}, w_{\text {Inter }}>w_{\mathrm{WH}}$ is maintained, our model is not sensitive to specific values of the weights of these terms. Specifically, 38 sets of weight parameters were tested and the transfer free energy scales were derived at residue A210 using each parameter set. The correlation coefficient $\left(\mathrm{R}^{2}\right)$ of the derived scales with the Moon-Fleming scale is 0.81 , with a small standard deviation of \pm 0.04 , indicating our model is robust with respect to the choice of weights. Previous studies have shown that the strength of a weak $\mathrm{H}$-bond is slightly stronger than half of that of a conventional H-bond, ${ }^{9-13}$ consistent with an assignment of $w_{\text {Inter }}>w_{\mathrm{WH}}$.

The energy of the TM region of an OMP in a specific configuration $\mathbf{d}$ is then calculated 
as the summation of strand energies of all TM strands:

$$
E(\mathbf{d})=\sum_{i} E(i ; \mathbf{d})
$$

\section{Calculating the folding free energy of the TM region of an OMP}

Considering each configuration $\mathbf{d}$ as a microstate, the partition function $Z_{\text {lipid }}$ of the TM region of an OMP in the lipid bilayer is then calculated by summing the Boltzmann factor of TM strands over all microstates:

$$
Z_{\text {lipid }}=\sum_{\mathbf{d} \in \Omega} e^{-\frac{E(\mathbf{d})}{k_{B} T}}
$$

The free energy of the protein in the lipid bilayer is then computed as:

$$
G_{\text {lipid }}=-k_{B} T \ln Z_{\text {lipid }}
$$

The folding free energy of the TM region of a protein is therefore:

$$
\Delta G=G_{\text {water }}-G_{\text {lipid }}
$$

where $G_{\text {water }}$ is assign a constant $C$. That is, the free energy of the ensemble of conformations of an OMP in the solution phase is not hugely affected by a point mutation. This assumption is reasonable, as OMPs in solution are unlikely to be in an extended linear form. For example,

a significant amount of residual structures were reported for denatured OmpX by NMR and MD studies, even under the strong condition of $8 \mathrm{M}$ urea. ${ }^{14,15}$

\section{Calculating transfer free energy scale of a host residue in OmpLA}

Following the work of reference, ${ }^{16}$ we take differences in the $\Delta G$ of a mutant at the host residue of OmpLA and the $\Delta G$ of Ala at the same host residue as the transfer free energy 
from water to bilayer $\Delta \Delta G$ of the mutated amino acid.

$$
\Delta \Delta G_{\text {mutant }}^{\text {Host }}=\Delta G^{\text {Host }}(\text { Ala })-\Delta G^{\text {Host }}(\text { mutant })
$$

We further simplify Equation 6 using Equation 5:

$$
\begin{aligned}
\Delta \Delta G_{\text {mutant }}^{\text {Host }} & =\Delta G^{\text {Host }}(\text { Ala })-\Delta G^{\text {Host }}(\text { mutant }) \\
& =\left[G_{\text {water,Ala }}^{\text {Host }}-G_{\text {lipid,Ala }}^{\text {Host }}\right]-\left[G_{\text {water,mutant }}^{\text {Host }}-G_{\text {lipid,mutant }}^{\text {Host }}\right] \\
& =G_{\text {lipid,mutant }}-G_{\text {lipid,Ala }},
\end{aligned}
$$

assuming $G_{\mathrm{water}, \mathrm{Ala}}^{\text {Host }}=G_{\mathrm{water}, \mathrm{mutant}}^{\mathrm{Host}}=C$. That is, the free energy of the ensemble of conformations of an OMP in the solution phase is not hugely affected by a point mutation. This assumption is reasonable as OMPs in solution are unlikely to be in an extended linear form. For example, a significant amount of residual structures were reported for denatured OmpX by NMR and MD studies, even under the strong condition of $8 \mathrm{M}$ urea. ${ }^{14,15}$

\section{Detecting transfer free energy scales with strong context depen- dency}

For $n_{i}$ number of host residues at the depth position $i$ whose transfer free energy scales have been calculated, we further calculate the pairwise correlation coefficients between scales

obtained for each of the the $\left(\begin{array}{c}n_{i} \\ 2\end{array}\right)$ pair of residues, and then take the average. We then exclude each of the $k$-th residue in turn and recalculate the average correlation among the $\left(\begin{array}{c}n_{i}-1 \\ 2\end{array}\right)$ scales. If this new averaged correlation coefficient increases by $\geq 10 \%$, we do not include this $k$-th residue in the calculation of the final averaged general scale. Therefore, both the average scale and the identification of deviating residues do not depend on the sequence in which residues were chosen for calculation. 


\section{Calculating depth-dependent transfer free energy profiles of amino acid residues}

All depth-dependent transfer free energy profiles $\left\{\Delta \Delta G_{a a(i)}\right\}$ of amino acid residues, except those for Tyr and Trp, are approximated by an asymmetric stepwise Gaussian function:

$$
\Delta \Delta G_{a a(i)}= \begin{cases}a_{0} e^{\left(-i^{2} / 2 a_{1}^{2}\right)}, & \text { if } i \geq 0 \\ a_{0} e^{\left(-i^{2} / 2 a_{2}^{2}\right)}, & \text { if } i<0\end{cases}
$$

where $i \in\{-p, \cdots, 0, \cdots, p\}$ denotes the position index of the TM residue, with $i=0$ corresponding to the center of the bilayer. We used $p=4$ in this study. For Trp and Tyr, a double Gaussian function is used:

$$
\Delta \Delta G_{a a(i)}=a_{0} e^{\left(-\left(i-a_{2}\right)^{2} / 2 a_{1}^{2}\right)}+a_{0} e^{\left(-\left(i+a_{4}\right)^{2} / 2 a_{3}^{2}\right)} .
$$

The fitted $\Delta \Delta G_{a a(i)}$ values and the values of $a_{0}, a_{1}, a_{2}, a_{3}, a_{4}$ can be found in Table S3.

\section{Comparing thermodynamic stability of OMPs in the asymmetric outer membrane and in the hypothetical symmetric outer mem- brane}

The thermodynamic stability of the TM region of an OMP is approximated by the total transfer free energy of the lipid-facing TM residues. Denote the amino acid residue in the $j$-th position on the $i$-th strand as $A_{i, j}$, the stability of an OMP in the asymmetric outer membrane is calculated as:

$$
\Delta \Delta G_{\text {asym }}=\sum_{i=1}^{N} \sum_{j=-p}^{p} \Delta \Delta G_{A_{i, j}(j)},
$$

where $N$ is the number of strands in the OMP, and $p$ is defined as above. 
The stability of an OMP in the hypothetical symmetric outer membrane is calculated as:

$$
\Delta \Delta G_{\mathrm{sym}}=\sum_{i=1}^{N} \sum_{j=-p}^{p} \Delta \Delta G_{A_{i, j}(j)}^{*},
$$

with

$$
\Delta \Delta G_{A_{i, j}(j)}^{*}= \begin{cases}\Delta \Delta G_{A_{i, j}(j)}, & \text { if } j \leq 0 \\ \Delta \Delta G_{A_{i, j}(-j)}, & \text { if } j>0\end{cases}
$$

By subtracting Equation 10 from Equation 11, we obtain the difference in thermodynamic stability between the TM region of an OMP in the native asymmetric outer membrane and in the hypothetical symmetric outer membrane.

\section{Comparing thermodynamic stabilities of OMPs in NC-IN and NC- OUT topologies}

Additive model. When an additive model is used, we calculate the thermodynamic stability of the TM region as the total transfer free energy of lipid-facing TM residues of a

given OMP. We denote $A_{i, j}$ as the amino acid in the $j$-th position of the $i$-th strand. The thermodynamic stability of an OMP in the NC-IN topology is calculated as:

$$
\Delta \Delta G_{\mathrm{NC}-\mathrm{IN}}=\sum_{i=1}^{N} \sum_{j=-p}^{p} \Delta \Delta G_{A_{i, j}(j)} \cdot \delta_{i j},
$$

with

$$
\delta_{i j}= \begin{cases}1, & \text { if } A_{i, j} \text { is lipid facing } \\ 0, & \text { if } A_{i, j} \text { is pore facing }\end{cases}
$$

Similarity, the thermodynamic stability of an OMP in NC-OUT topology is calculated as:

$$
\Delta \Delta G_{\mathrm{NC}-\mathrm{OUT}}=\sum_{i=1}^{N} \sum_{j=-p}^{p} \Delta \Delta G_{A_{i, j}(-j)} \cdot \delta_{i j},
$$


with

$$
\delta_{i j}= \begin{cases}1, & \text { if } A_{i, j} \text { is lipid facing } \\ 0 . & \text { if } A_{i, j} \text { is pore facing. }\end{cases}
$$

By subtracting Equation 13 from Equation 15, we obtain the difference in stability when adopting the two different topologies:

$$
\begin{aligned}
\Delta \Delta G_{\mathrm{topo}} & =\Delta \Delta G_{\mathrm{NC}-\mathrm{OUT}}-\Delta \Delta G_{\mathrm{NC}-\mathrm{IN}} \\
& = \begin{cases}\geq 0, & \text { OMPs in NC-IN topology } \\
<0, & \text { OMPs in NC-OUT topology }\end{cases}
\end{aligned}
$$

The predicted topology is chosen to be the one with lower $\Delta \Delta G$.

Nonadditive model. Alternatively, we directly calculate the folding free energies of the TM region of an OMP using Equation 5 by directly summing up the Boltzman factors of residues in the TM regions over all enumerated miscrostates. The difference in thermodynamic stability $\Delta \Delta G_{\text {topo }}$ between the two topologies is then:

$$
\begin{aligned}
\Delta \Delta G_{\mathrm{topo}} & =\Delta G_{\mathrm{NC}-\mathrm{OUT}}-\Delta G_{\mathrm{NC}-\mathrm{IN}} \\
& = \begin{cases}\geq 0, & \text { OMPs in NC-IN topology } \\
<0, & \text { OMPs in NC-OUT topology }\end{cases}
\end{aligned}
$$

\section{Calculating cooperativity of residue pairs}

The cooperativity of two residues at the positions (WT, WT) of a pair of host positions in OmpLA is calculated as: ${ }^{16}$ 


$$
\Delta \Delta \Delta G_{\text {mutant }}^{\text {Host Pair }}=\Delta \Delta G_{(\text {WT, mutant })}^{\text {Host Pair }}+\Delta \Delta G_{(\text {mutant }, \mathrm{WT})}^{\text {Host Pair }}-\Delta \Delta G_{(\text {mutant, mutant })}^{\text {Host Pair }} \cdot
$$

\section{References}

(1) Naveed, H.; Jackups, R., Jr.; Liang, J. Proc Natl Acad Sci U S A 2009, 106, 1273512740.

(2) Wouters, M. A.; Curmi, P. M. Proteins 1995, 22, 119-131.

(3) Ho, B.; Curmi, P. J Mol Biol 2002, 317, 291-308.

(4) Jackups, R., Jr.; Liang, J. J Mol Biol 2005, 354, 979-993.

(5) Schrödinger, LLC,

(6) Kabsch, W.; Sander, C. Biopolymers 1983, 22, 2577-2637.

(7) Lomize, M. A.; Lomize, A. L.; Pogozheva, I. D.; Mosberg, H. I. Bioinformatics 2006, 22, 623-625.

(8) Miyazawa, S.; Jernigan, R. J Mol Biol 1996, 256, 623-644.

(9) Vargas, R.; Garza, J.; Dixon, D. A.; Hay, B. P. Journal of the American Chemical Society 2000, 122.

(10) Scheiner, S.; Kar, T.; Gu, Y. J. Biol. Chem. 2001, 276, 9832-9837.

(11) Scheiner, S. J Phys Chem B 2005, 109, 16132-16141.

(12) Scheiner, S. J Phys Chem B 2006, 110, 18670-18679.

(13) Bowie, J. U. Curr. Opin. Struct. Biol. 2011, 21, 42-49. 
(14) Tafer, H.; Hiller, S.; Hilty, C.; Fernandez, C.; Wuthrich, K. Biochemistry 2004, 43, 860-869.

(15) Krautler, V.; Hiller, S.; Hunenberger, P. Eur Biophys J 2010, 39, 1421-1432.

(16) Moon, C.; Fleming, K. Proc Natl Acad Sci U S A 2011, 108, 10174-10177.

(17) Kingma, R.; Fragiathaki, M.; Snijder, H.; Dijkstra, B.; Verheij, H.; Dekker, N.; Egmond, M. Biochemistry 2000, 39, 10017-10022.

(18) Li, X.; Hu, C.; Liang, J. Proteins 2003, 53, 792-805.

(19) Hsieh, D.; Davis, A.; Nanda, V. Protein Sci. 2012, 21, 50-62.

(20) Slusky, J.; Dunbrack, R., Jr. Bioinformatics 2013, 29, 2122-2128.

(21) Hessa, T.; Kim, H.; Bihlmaier, K.; Lundin, C.; Boekel, J.; Andersson, H.; Nilsson, I.; White, S. H.; von Heijne, G. Nature 2005, 433, 377-381.

(22) Wimley, W. C.; Creamer, T. P.; White, S. H. Biochemistry 1996, 35, 5109-5124. 


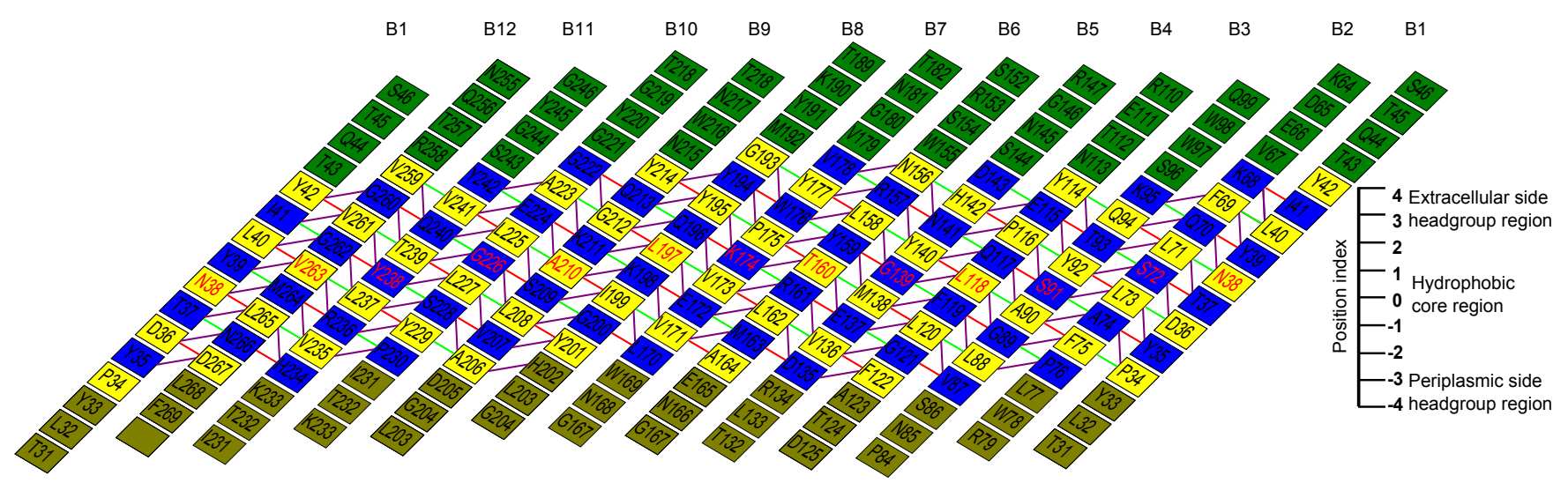

Fig. S1. The discrete model of the transmembrane region of outer membrane phospholipase A (OmpLA). In this model, a $\beta$-strand has 16 residues: 4 residues in the extracellular cap region (green), 9 residues in the TM region (pore-facing residues in blue, lipid-facing residues in yellow), and 3 residues in the periplasmic cap region (olive). Position index $i$ of TM residues as integers are listed on the vertical line. Residues at position $i=0$ are highlighted in red. Important inter-strand interactions between two neighboring TM residues are drawn in red (strong H-bond), green (non H-bond), and brown (weak H-bond) lines. 

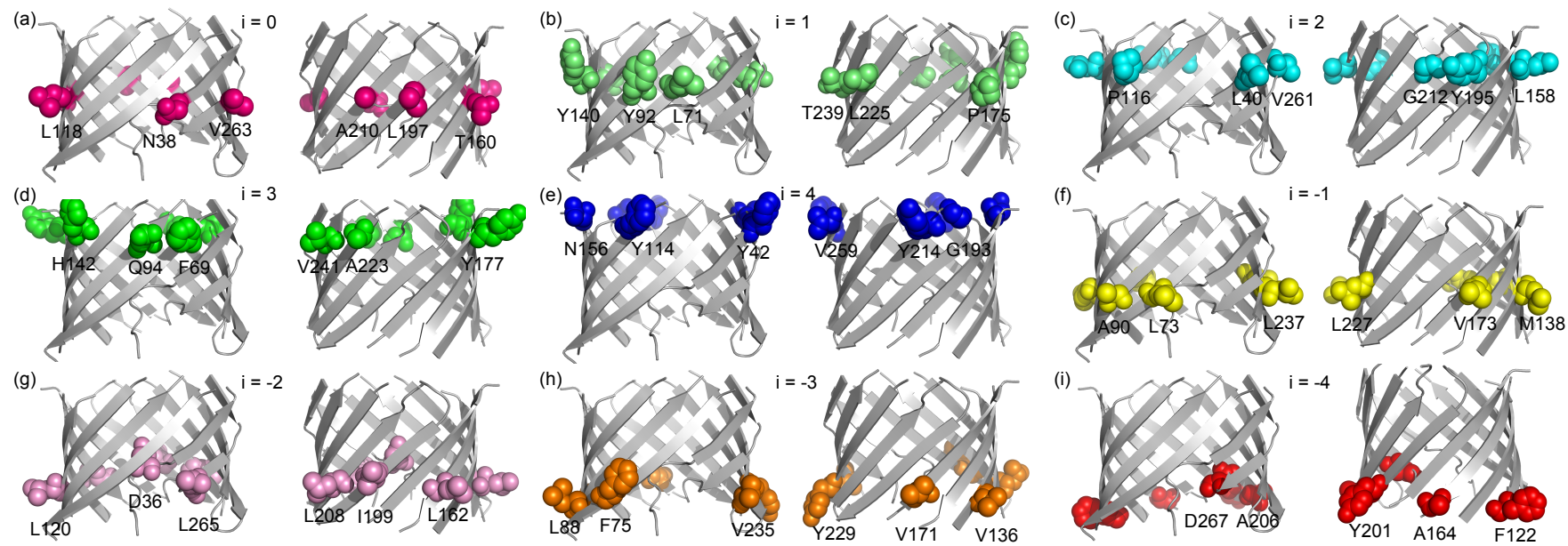

Fig. S2. 53 lipid-facing TM residues are used in deriving transfer free energy scales in OmpLA. For depth-position $i$, lipid-facing residues with the same position index $i$ are used as host residues. Side-chains of the 53 host residues are shown in spheres. 


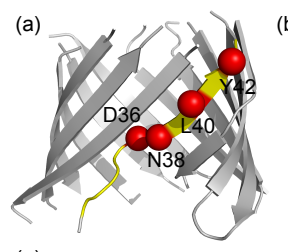

(e)
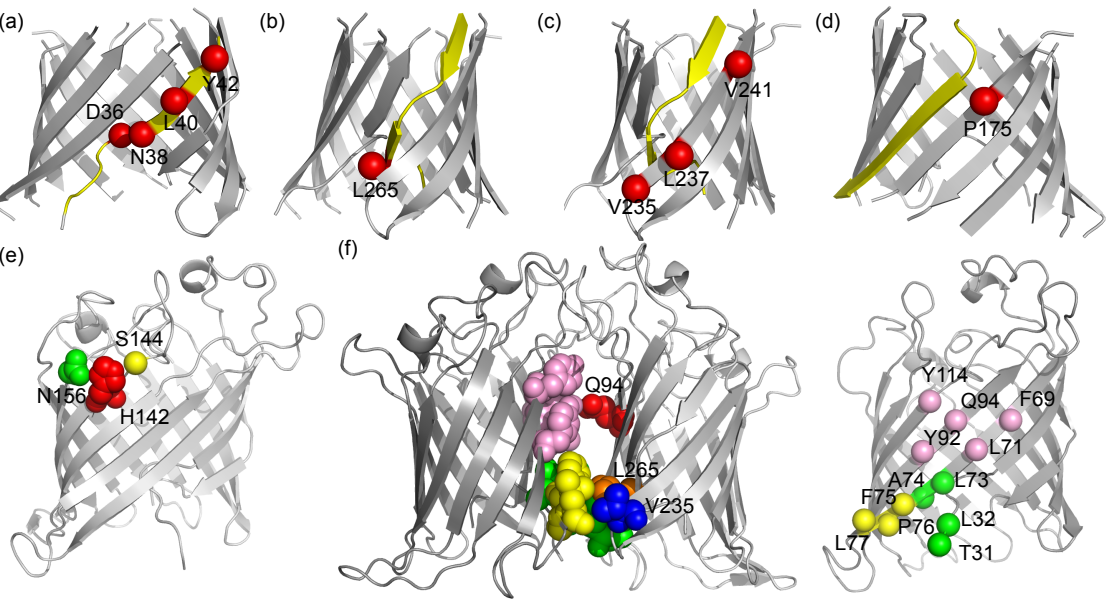

Fig. S3. Host residues demonstrating strong context dependency in transfer free energy scales are located in structurally deformed environment (a-d), active-site residues (e), or forming inter-molecular interactions for dimerization (f). (a) D36, N38, L40, and Y42 are located in $\beta 1$, which contains a TM coil (residue 35-38). (b) L265 is located in $\beta 12$, which also contains a TM coil (residue 260-263) (c) V235, L237, and V241 are next to a deformed strand $\beta 12$ as their neighboring strand. (d) P175 in $\beta 7$ is neighboring the deformed portion of strand $\beta 8$. (e) H142 (red) and N156 (green) are part of the catalytic triad (H142-S144N156) of OmpLA for its enzymatic function. ${ }^{17}$ (f) Q94 (red), V235 (blue), and L265 (orange) provide inter-molecular interactions for the OmpLA dimerization: Q94 makes contacts with F69, L71, Y92, and Y114 (pink); V235 with F75, P76, and L77 (yellow); L265 with T31, L32, L73, A74, F75, P76, and L77 (yellow and green). Inter-molecule contacts are identified using a simplicial edge criteria ${ }^{18}$ 
(a)

\begin{tabular}{|c|c|c|c|}
\hline & $\Delta \Delta \mathrm{G}_{\mathrm{w}, \mathrm{I}}^{\mathrm{O}}$ & $\Delta \Delta \mathrm{G}^{\mathrm{aa}}{ }_{\text {app }}$ & $\Delta \Delta \mathrm{G}_{\text {Octanol }}$ \\
\hline$E z \beta^{a}$ & $N A^{c} / N A^{d} / 0.65^{e}$ & NA / NA / 0.84 & NA / NA / 0.55 \\
\hline Slusky-Dunbrack & NA $/ 0.31 / 0.30$ & NA / $0.44 / 0.43$ & NA / $0.52 / 0.51$ \\
\hline$E_{\mathrm{b}}$ & $0.47 / 0.64 / 0.64$ & $0.45 / 0.78 / 0.76$ & $0.50 / 0.68 / 0.68$ \\
\hline$\Delta \Delta G^{\mathrm{A} 210}$ & $0.81 / 0.81 / 0.81$ & $0.86 / 0.88 / 0.89$ & $0.67 / 0.67 / 0.66$ \\
\hline
\end{tabular}

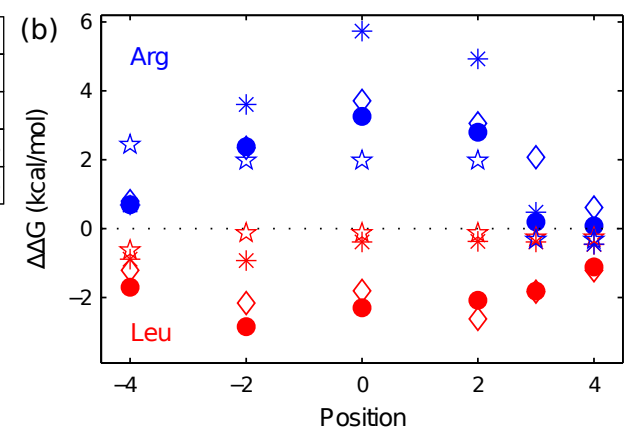

Fig. S4. (a) Three scales based on probabilities of finding residues in the TM strands (values see Table S6), $E z \beta,{ }^{19}$ Slusky-Dunbrack scale, ${ }^{20}$ and $E_{b},{ }^{4}$ were compared with three experimental hydrophobicity scales. (b) Computed transfer free energies (closed circle) of Arg and Leu agree well with the experimentally measured values (diamond). ${ }^{16}$ However, profiles of empirical energy values either from statistical distribution (empty star) ${ }^{4}$ or from the earlier empirical energy function (asterisk) ${ }^{1}$ were unable to reproduce the experimental values (diamond). 


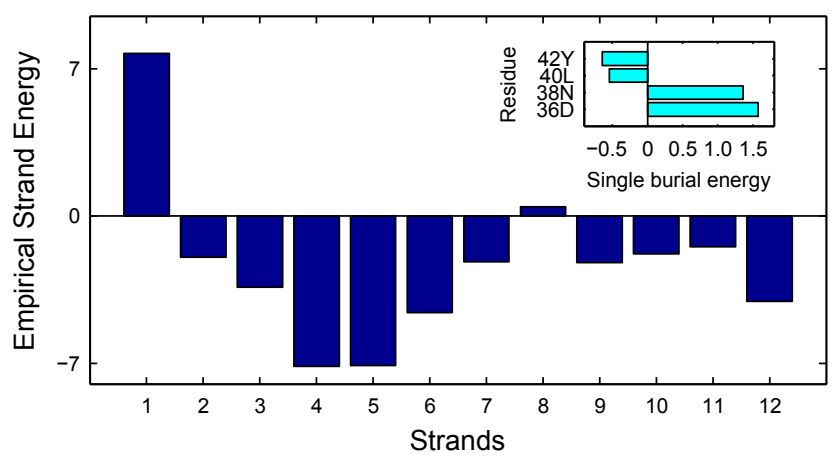

Fig. S5. Strand stability analysis of OmpLA detects unstable strand $\beta 1$ and its unstable residues. Strand energy of each TM strand of the native structure is calculated based on ref ${ }^{1}$ . Strand $\beta 1$ is a relatively unstable TM strand, as it has significantly higher strand energy than other TM strands. Lipid-facing residues D36 and N38 are detected as the unstable residues of strand $\beta 1$ based on their high single burial energy (inset figure). 

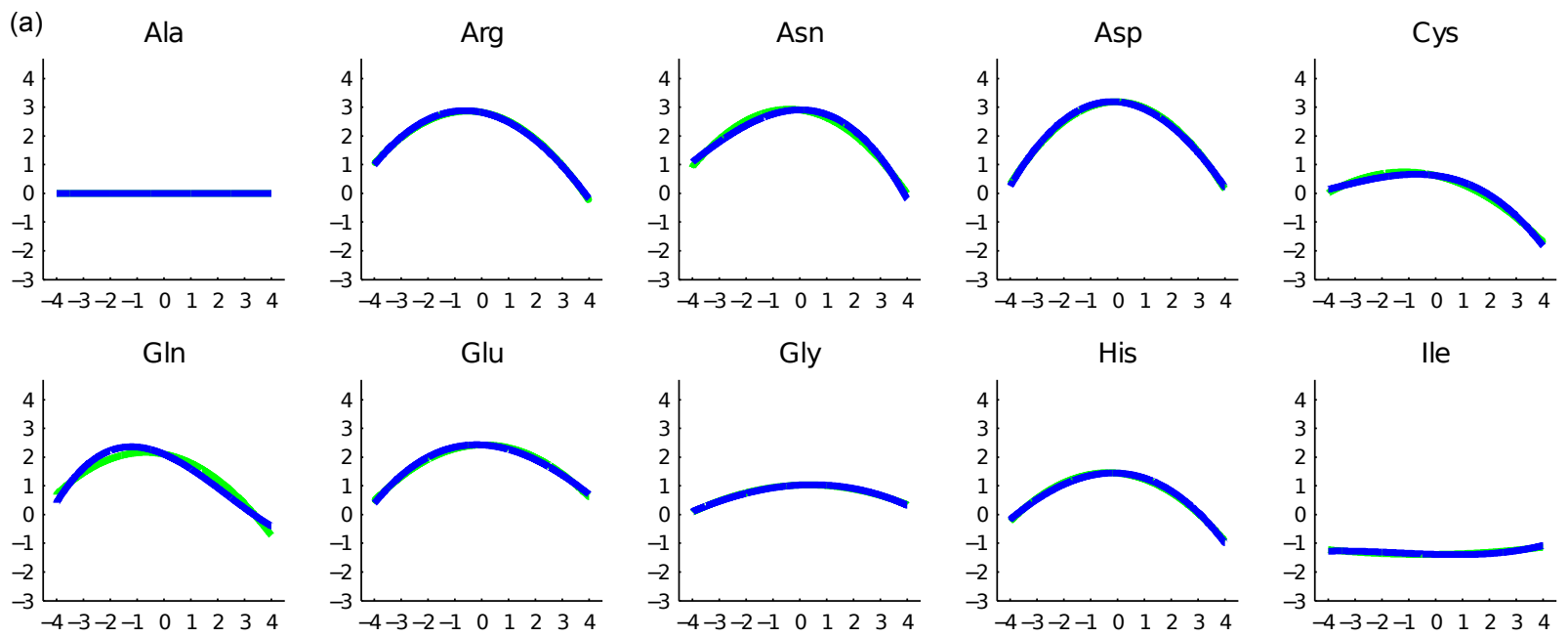

lle
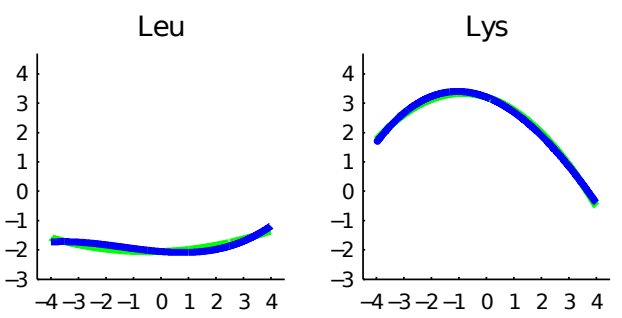

Met

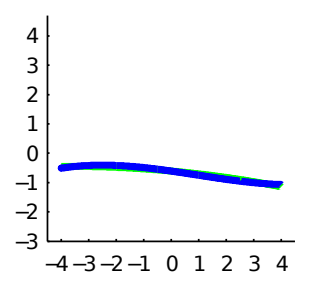

Phe
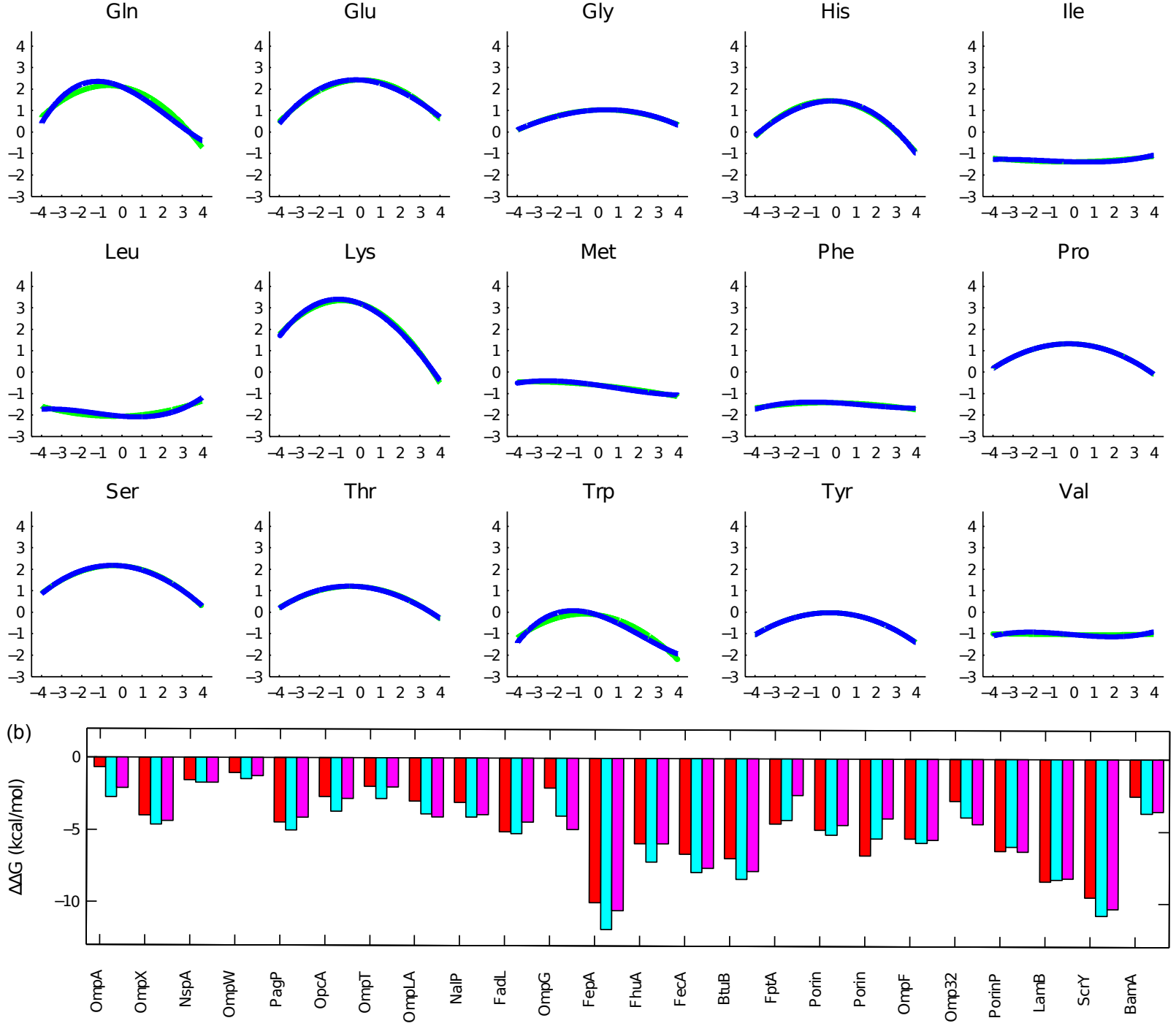

Fig S6. Approximation on thermodynamic stability is not sensitive to parameters of depthdependent profiles. (a) Polynomial functions were used to derive depth-dependent transfer free energy profiles. Asymmetry in the profiles was observed in both second-degree (green) and third-degree (blue) polynomial function. (b) The stability differences of 24 OMPs in the asymmetric and a symmetric OM were determined using profiles derived from asymmetric Gaussian (red), second-degree polynomial (cyan), and third-degree polynomial (blue). All OMPs showed higher stability $(\Delta \Delta G<0)$ in the asymmetric OM. 


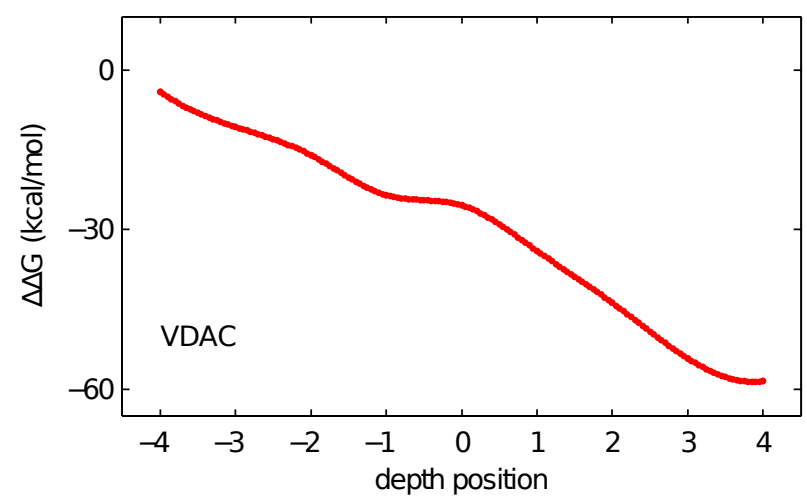

Fig. S7. Spontaneous insertion is predicted for mitochondria outer membrane protein VDAC. Folding energy of VDAC (PDB id: 3EMN) is approximated as the total transfer free energy of lipid-facing residues inserted into the bilayer. No energy barrier is observed during the folding process. 
Table S1. Transfer free energy $\Delta \Delta \mathrm{G}^{\text {host }}(\mathrm{kcal} / \mathrm{mol})$ at different host residues of OmpLA and the general transfer free energy $\Delta \Delta G_{(i)}$ calculated from non-context dependent hosts at each depth-position $i$

\begin{tabular}{|c|c|c|c|c|c|c|}
\hline \multirow[t]{2}{*}{ A. A } & \multicolumn{5}{|c|}{$\Delta \Delta G(\mathrm{kcal} / \mathrm{mol})$} & \multirow[b]{2}{*}{$\Delta \Delta G_{(-4)}$} \\
\hline & $\Delta \Delta G^{\mathrm{F} 122}$ & $\Delta \Delta G^{\mathrm{A} 164}$ & $\Delta \Delta G^{\mathrm{Y} 201}$ & $\Delta \Delta G^{\mathrm{A} 206}$ & $\Delta \Delta G^{\mathrm{D} 267}$ & \\
\hline A & 0 & 0 & 0 & 0 & 0 & $0 \pm 0$ \\
\hline $\mathrm{R}$ & 1.86 & 0.69 & 1.16 & 0.71 & 1.12 & $1.11 \pm 0.21$ \\
\hline $\mathrm{N}$ & 1.87 & 1.03 & 0.85 & 1.45 & 1.14 & $1.27 \pm 0.18$ \\
\hline $\mathrm{D}$ & 1.07 & 0.30 & 0.03 & -0.4 & -0.12 & $0.18 \pm 0.25$ \\
\hline $\mathrm{C}$ & 0.25 & 0.12 & 0.07 & 0.03 & 0.12 & $0.12 \pm 0.04$ \\
\hline Q & 0.82 & 0.57 & 0.30 & 0.73 & 0.36 & $0.56 \pm 0.10$ \\
\hline $\mathrm{E}$ & 1.14 & 0.14 & 0.24 & 0.71 & 0.42 & $0.53 \pm 0.18$ \\
\hline G & 0.62 & 0.12 & 0.25 & -0.01 & -0.03 & $0.19 \pm 0.12$ \\
\hline $\mathrm{H}$ & 0.20 & -0.47 & -0.32 & 0.62 & 0.22 & $0.05 \pm 0.20$ \\
\hline I & -1.70 & -1.14 & -1.33 & -0.65 & -1.74 & $-1.31 \pm 0.20$ \\
\hline L & -1.63 & -1.70 & -1.57 & -1.78 & -1.80 & $-1.70 \pm 0.04$ \\
\hline K & 2.13 & 1.89 & 2.08 & 2.18 & 2.24 & $2.10 \pm 0.06$ \\
\hline M & -0.48 & -0.38 & -0.69 & -0.48 & -0.57 & $-0.52 \pm 0.05$ \\
\hline $\mathrm{F}$ & -2.17 & -2.01 & -1.40 & -1.63 & -1.85 & $-1.81 \pm 0.14$ \\
\hline $\mathrm{P}$ & -0.37 & 0.43 & 0.41 & 0.36 & -0.49 & $0.07 \pm 0.20$ \\
\hline$S$ & 1.75 & 0.90 & 0.76 & 0.24 & 1.36 & $1.00 \pm 0.26$ \\
\hline $\mathrm{T}$ & 0.29 & 0.28 & 0.23 & -0.43 & 0.69 & $0.21 \pm 0.18$ \\
\hline W & -1.86 & -1.59 & -1.53 & -0.50 & -1.37 & $-1.37 \pm 0.23$ \\
\hline Y & -1.20 & -1.10 & -1.10 & -1.00 & -0.78 & $-1.04 \pm 0.07$ \\
\hline V & -1.33 & -0.84 & -0.7 & -1.15 & -1.29 & $-1.06 \pm 0.12$ \\
\hline
\end{tabular}

\begin{tabular}{rrrrrrrc} 
& $\Delta \Delta G^{\mathrm{V} 235}$ & $\Delta \Delta G^{\mathrm{F} 75}$ & $\Delta \Delta G^{\mathrm{L} 88}$ & $\Delta \Delta G^{\mathrm{V} 136}$ & $\Delta \Delta G^{\mathrm{V} 171}$ & $\Delta \Delta G^{\mathrm{Y} 229}$ & $\Delta \Delta G_{(-3)}$ \\
\hline A & 0 & 0 & 0 & 0 & 0 & 0 & $0 \pm 0$ \\
\hline
\end{tabular}


Table S1 continued

\begin{tabular}{|c|c|c|c|c|c|c|c|}
\hline \multirow{2}{*}{$\frac{A \cdot A}{R}$} & \multicolumn{7}{|c|}{$\Delta \Delta G(\mathrm{kcal} / \mathrm{mol})$} \\
\hline & -0.66 & 0.67 & 1.4 & 3.27 & 1.41 & 1.70 & $1.69 \pm 0.43$ \\
\hline $\mathrm{N}$ & 0.34 & 0.33 & 1.06 & 2.27 & 1.59 & 1.49 & $1.35 \pm 0.32$ \\
\hline $\mathrm{D}$ & 1.10 & 0.21 & 0.72 & 3.17 & 3.17 & 1.31 & $1.72 \pm 0.62$ \\
\hline $\mathrm{C}$ & 0.28 & 0.15 & 0.22 & 0.81 & 0.83 & 0.26 & $0.45 \pm 0.15$ \\
\hline $\mathrm{Q}$ & -0.13 & 0.79 & 0.41 & 0.58 & 2.22 & 2.15 & $1.23 \pm 0.39$ \\
\hline $\mathrm{E}$ & -0.72 & 0.40 & 0.39 & 1.87 & 1.07 & 1.50 & $1.05 \pm 0.29$ \\
\hline G & -0.32 & 0.26 & -0.02 & 0.08 & 0.29 & 0.80 & $0.28 \pm 0.14$ \\
\hline $\mathrm{H}$ & -0.90 & 0.20 & 0.79 & -0.26 & 0.09 & 0.67 & $0.30 \pm 0.19$ \\
\hline I & -0.31 & -1.41 & -1.15 & -0.57 & -0.65 & -1.76 & $-1.11 \pm 0.23$ \\
\hline $\mathrm{L}$ & -0.61 & -2.16 & -2.95 & -1.06 & -0.11 & -2.25 & $-1.71 \pm 0.50$ \\
\hline K & 0.84 & 0.60 & 1.67 & 2.02 & 2.39 & 1.89 & $1.71 \pm 0.30$ \\
\hline M & -0.39 & -1.27 & -1.58 & 0.64 & 0.61 & -0.27 & $-0.37 \pm 0.46$ \\
\hline $\mathrm{F}$ & -0.46 & -1.00 & -2.42 & -0.77 & -0.26 & -2.11 & $-1.31 \pm 0.41$ \\
\hline $\mathrm{P}$ & 0.45 & -0.02 & 0.94 & 0.33 & 1.76 & 0.75 & $0.75 \pm 0.30$ \\
\hline S & -0.16 & 0.59 & 0.59 & 1.33 & 1.08 & 1.81 & $1.08 \pm 0.23$ \\
\hline $\mathrm{T}$ & -0.05 & 0.15 & 0.86 & 0.57 & 0.32 & 1.17 & $0.61 \pm 0.18$ \\
\hline W & -0.34 & -0.23 & -1.41 & -0.59 & -0.29 & -0.63 & $-0.63 \pm 0.21$ \\
\hline Y & -0.74 & -0.48 & -1.64 & -0.16 & -0.80 & -0.17 & $-0.65 \pm 0.27$ \\
\hline V & -0.45 & -0.96 & -0.92 & -0.75 & -0.70 & -1.46 & $-0.96 \pm 0.13$ \\
\hline
\end{tabular}

\begin{tabular}{cccccccc} 
& $\Delta \Delta G^{\mathrm{D} 36}$ & $\Delta \Delta G^{\mathrm{L} 265}$ & $\Delta \Delta G^{\mathrm{L} 120}$ & $\Delta \Delta G^{\mathrm{L} 162}$ & $\Delta \Delta G^{\mathrm{I} 199}$ & $\Delta \Delta G^{\mathrm{L} 208}$ & $\Delta \Delta G_{(-2)}$ \\
\hline $\mathrm{A}$ & 0 & 0 & 0 & 0 & 0 & 0 & $0 \pm 0$ \\
$\mathrm{R}$ & -0.24 & 1.49 & 2.38 & 4.04 & 2.56 & 2.36 & $2.84 \pm 0.40$ \\
$\mathrm{~N}$ & 0.06 & 0.72 & 2.04 & 4.62 & 3.11 & 1.66 & $2.86 \pm 0.66$ \\
$\mathrm{D}$ & 0.51 & 0.28 & 2.12 & 4.47 & 3.15 & 2.38 & $3.03 \pm 0.53$ \\
\hline
\end{tabular}


Table S1 continued

\begin{tabular}{|c|c|c|c|c|c|c|c|}
\hline \multirow{2}{*}{$\frac{A \cdot A}{C}$} & \multicolumn{7}{|c|}{$\Delta \Delta G(\mathrm{kcal} / \mathrm{mol})$} \\
\hline & 0.37 & 1.02 & -0.34 & 0.82 & 0.60 & 0.41 & $0.37 \pm 0.25$ \\
\hline Q & -0.37 & 1.30 & 1.87 & 4.83 & 3.40 & 1.34 & $2.86 \pm 0.79$ \\
\hline $\mathrm{E}$ & -0.03 & 0.17 & 1.89 & 3.71 & 2.82 & 1.28 & $2.42 \pm 0.53$ \\
\hline G & -0.96 & 0.42 & 0.33 & 1.11 & 0.92 & 0.58 & $0.74 \pm 0.17$ \\
\hline $\mathrm{H}$ & -0.65 & 1.23 & -0.05 & 1.27 & 0.47 & 1.22 & $0.73 \pm 0.32$ \\
\hline I & 0.28 & -0.97 & -2.02 & -2.00 & -1.73 & -1.42 & $-1.79 \pm 0.14$ \\
\hline $\mathrm{L}$ & -0.52 & -1.36 & -2.85 & -1.89 & -2.02 & -2.27 & $-2.26 \pm 0.21$ \\
\hline K & -0.09 & 1.83 & 4.36 & 4.82 & 3.67 & 2.46 & $3.83 \pm 0.51$ \\
\hline M & -0.28 & 0.48 & -1.29 & 0.06 & 0.24 & -0.46 & $-0.36 \pm 0.34$ \\
\hline $\mathrm{F}$ & -0.24 & -1.53 & -1.47 & -1.96 & -1.74 & -1.59 & $-1.69 \pm 0.11$ \\
\hline $\mathrm{P}$ & 1.07 & 0.02 & 2.70 & 0.96 & 1.99 & 0.89 & $1.64 \pm 0.44$ \\
\hline S & -0.49 & 1.43 & 2.06 & 4.01 & 2.70 & 1.20 & $2.49 \pm 0.59$ \\
\hline $\mathrm{T}$ & -0.21 & 0.80 & 1.31 & 1.39 & 1.52 & 1.04 & $1.32 \pm 0.10$ \\
\hline W & -0.51 & 0.14 & 0.25 & 0.16 & 0.19 & -0.24 & $0.09 \pm 0.11$ \\
\hline Y & -0.30 & 0.09 & -0.41 & -0.15 & -0.25 & 0.49 & $-0.08 \pm 0.20$ \\
\hline V & -0.29 & -1.07 & -1.43 & -1.34 & -1.40 & -0.92 & $-1.27 \pm 0.12$ \\
\hline
\end{tabular}

\begin{tabular}{cccccccc} 
& $\Delta \Delta G^{\mathrm{L} 237}$ & $\Delta \Delta G^{\mathrm{L} 73}$ & $\Delta \Delta G^{\mathrm{A} 90}$ & $\Delta \Delta G^{\mathrm{M} 138}$ & $\Delta \Delta G^{\mathrm{V} 173}$ & $\Delta \Delta G^{\mathrm{L} 227}$ & $\Delta \Delta G_{(-1)}$ \\
\hline $\mathrm{A}$ & 0 & 0 & 0 & 0 & 0 & 0.00 & $0 \pm 0$ \\
$\mathrm{R}$ & 1.98 & 2.28 & 3.43 & 1.76 & 4.47 & 1.53 & $2.69 \pm 0.55$ \\
$\mathrm{~N}$ & 2.34 & 1.96 & 3.56 & 1.06 & 3.14 & 1.71 & $2.29 \pm 0.46$ \\
$\mathrm{D}$ & 1.82 & 1.86 & 3.51 & 1.53 & 3.72 & 1.19 & $2.36 \pm 0.52$ \\
$\mathrm{C}$ & 1.25 & 0.27 & 1.31 & 0.81 & 1.52 & 0.01 & $0.78 \pm 0.29$ \\
$\mathrm{Q}$ & 2.44 & 1.60 & 2.01 & 0.70 & 2.13 & 1.37 & $1.56 \pm 0.26$ \\
$\mathrm{E}$ & 1.54 & 1.54 & 3.08 & 0.66 & 2.65 & 1.33 & $1.85 \pm 0.44$ \\
\hline
\end{tabular}


Table S1 continued

\begin{tabular}{|c|c|c|c|c|c|c|c|}
\hline \multirow{2}{*}{$\frac{A \cdot A}{G}$} & \multicolumn{7}{|c|}{$\Delta \Delta G(k \mathrm{cal} / \mathrm{mol})$} \\
\hline & 0.17 & 1.17 & 2.05 & 0.20 & 1.24 & 0.49 & $1.03 \pm 0.32$ \\
\hline $\mathrm{H}$ & 1.87 & 0.38 & 1.27 & 0.87 & 1.96 & 0.67 & $1.03 \pm 0.27$ \\
\hline$I$ & 0.76 & -1.60 & -1.38 & -0.08 & -0.02 & -1.06 & $-0.83 \pm 0.33$ \\
\hline L & -0.10 & -2.04 & -2.20 & -0.14 & -0.35 & -2.68 & $-1.48 \pm 0.52$ \\
\hline K & 2.42 & 2.78 & 2.98 & 2.05 & 4.15 & 2.46 & $2.88 \pm 0.35$ \\
\hline M & 1.22 & -1.18 & -1.85 & -0.42 & 0.70 & -1.46 & $-0.84 \pm 0.45$ \\
\hline $\mathrm{F}$ & 0.64 & -1.63 & -2.07 & -0.02 & 0.22 & -2.01 & $-1.10 \pm 0.50$ \\
\hline $\mathrm{P}$ & 0.73 & -0.08 & 2.07 & 0.12 & 0.34 & 1.09 & $0.71 \pm 0.39$ \\
\hline$S$ & 1.31 & 1.65 & 2.97 & 0.64 & 2.17 & 1.30 & $1.75 \pm 0.39$ \\
\hline $\mathrm{T}$ & 0.42 & 0.94 & 1.87 & -0.35 & 1.33 & 0.50 & $0.86 \pm 0.38$ \\
\hline W & 1.87 & -0.81 & 0.04 & 0.84 & 0.75 & -1.16 & $-0.07 \pm 0.40$ \\
\hline $\mathrm{Y}$ & 0.50 & -0.57 & 0.33 & 0.11 & 0.49 & -0.71 & $-0.07 \pm 0.24$ \\
\hline V & -0.09 & -1.62 & -1.22 & 0.39 & -0.16 & -0.43 & $-0.61 \pm 0.36$ \\
\hline
\end{tabular}

\begin{tabular}{cccccccc} 
& $\Delta \Delta G^{\mathrm{N} 38}$ & $\Delta \Delta G^{\mathrm{L} 118}$ & $\Delta \Delta G^{\mathrm{T} 160}$ & $\Delta \Delta G^{\mathrm{L} 197}$ & $\Delta \Delta G^{\mathrm{A} 210}$ & $\Delta \Delta G^{\mathrm{V} 263}$ & $\Delta \Delta G_{(0)}$ \\
\hline $\mathrm{A}$ & 0 & 0 & 0 & 0 & 0 & 0 & $0 \pm 0$ \\
$\mathrm{R}$ & 1.04 & 3.29 & 5.08 & 1.76 & 3.26 & 2.08 & $3.09 \pm 0.58$ \\
$\mathrm{~N}$ & 0.87 & 3.96 & 3.54 & 3.74 & 3.10 & 2.88 & $3.44 \pm 0.20$ \\
$\mathrm{D}$ & 1.14 & 4.30 & 3.78 & 1.83 & 4.04 & 3.89 & $3.57 \pm 0.44$ \\
$\mathrm{C}$ & 0.85 & 0.57 & 0.47 & 0.64 & 0.38 & 0.91 & $0.59 \pm 0.09$ \\
$\mathrm{Q}$ & 0.28 & 3.67 & 2.89 & 2.85 & 2.25 & 2.56 & $2.84 \pm 0.24$ \\
$\mathrm{E}$ & 0.60 & 3.35 & 3.00 & 3.41 & 2.89 & 1.76 & $2.88 \pm 0.30$ \\
$\mathrm{G}$ & -0.06 & 1.05 & 0.88 & 1.20 & 1.15 & 1.32 & $1.12 \pm 0.07$ \\
$\mathrm{H}$ & 0.83 & 2.82 & 3.04 & 2.00 & 3.23 & 2.43 & $2.70 \pm 0.22$ \\
$\mathrm{I}$ & 0.50 & -2.25 & -1.30 & -1.89 & -1.53 & -1.27 & $-1.65 \pm 0.19$ \\
\hline
\end{tabular}


Table S1 continued

\begin{tabular}{|c|c|c|c|c|c|c|c|}
\hline \multirow{2}{*}{$\frac{\mathrm{A} \cdot \mathrm{A}}{\mathrm{L}}$} & \multicolumn{7}{|c|}{$\Delta \Delta G(\mathrm{kcal} / \mathrm{mol})$} \\
\hline & 0.15 & -2.47 & -2.46 & -2.25 & -2.30 & -2.49 & $-2.39 \pm 0.05$ \\
\hline K & 0.16 & 4.60 & 6.88 & 4.57 & 3.97 & 2.15 & $4.43 \pm 0.76$ \\
\hline $\mathrm{M}$ & 0.18 & -0.50 & -0.18 & 0.48 & -0.62 & -0.45 & $-0.25 \pm 0.20$ \\
\hline $\mathrm{F}$ & 0.54 & -1.79 & -2.28 & -2.24 & -1.82 & -1.65 & $-1.96 \pm 0.13$ \\
\hline $\mathrm{P}$ & 0.26 & 1.22 & 1.61 & 1.46 & 1.81 & 0.92 & $1.40 \pm 0.15$ \\
\hline S & 0.47 & 3.19 & 3.14 & 2.14 & 1.73 & 2.73 & $2.59 \pm 0.28$ \\
\hline $\mathrm{T}$ & 0.20 & 1.75 & 1.46 & 1.61 & 1.07 & 1.11 & $1.40 \pm 0.13$ \\
\hline $\mathrm{W}$ & 0.35 & -0.69 & 1.37 & 0.22 & -0.34 & -0.23 & $0.07 \pm 0.36$ \\
\hline Y & 0.25 & -0.09 & -0.36 & -0.41 & -0.45 & 0.42 & $-0.18 \pm 0.16$ \\
\hline $\mathrm{V}$ & 0.52 & -1.57 & -0.11 & -1.42 & -1.39 & -1.52 & $-1.20 \pm 0.27$ \\
\hline
\end{tabular}

\begin{tabular}{cccccccc} 
& $\Delta \Delta G^{\mathrm{P} 175}$ & $\Delta \Delta G^{\mathrm{L} 71}$ & $\Delta \Delta G^{\mathrm{Y} 92}$ & $\Delta \Delta G^{\mathrm{Y} 140}$ & $\Delta \Delta G^{\mathrm{L} 225}$ & $\Delta \Delta G^{\mathrm{T} 239}$ & $\Delta \Delta G_{(1)}$ \\
\hline $\mathrm{A}$ & 0 & 0 & 0 & 0 & 0 & 0 & $0 \pm 0$ \\
$\mathrm{R}$ & 1.15 & 2.56 & 1.26 & 3.51 & 1.77 & 2.47 & $2.31 \pm 0.38$ \\
$\mathrm{~N}$ & 1.96 & 3.01 & 1.61 & 2.90 & 2.46 & 2.70 & $2.54 \pm 0.25$ \\
$\mathrm{D}$ & 1.54 & 2.83 & 1.90 & 3.54 & 2.71 & 2.62 & $2.72 \pm 0.26$ \\
$\mathrm{C}$ & 0.87 & 0.03 & -0.61 & 1.27 & -0.05 & 0.92 & $0.31 \pm 0.34$ \\
$\mathrm{Q}$ & 0.98 & 1.53 & 1.19 & 2.06 & 0.23 & 2.84 & $1.57 \pm 0.44$ \\
$\mathrm{E}$ & 0.72 & 2.80 & 2.30 & 2.57 & 1.84 & 2.10 & $2.32 \pm 0.17$ \\
$\mathrm{G}$ & 0.78 & 0.78 & 1.14 & 1.24 & 1.05 & 0.43 & $0.93 \pm 0.15$ \\
$\mathrm{H}$ & 1.82 & 0.37 & 0.11 & 1.88 & -0.18 & 1.74 & $0.78 \pm 0.43$ \\
$\mathrm{I}$ & -0.09 & -2.22 & -0.93 & -0.02 & -2.39 & -1.64 & $-1.44 \pm 0.44$ \\
$\mathrm{~L}$ & -0.02 & -2.84 & -1.24 & -0.36 & -2.08 & -1.77 & $-1.66 \pm 0.42$ \\
$\mathrm{~K}$ & 1.48 & 2.23 & 1.69 & 3.37 & 0.98 & 2.86 & $2.23 \pm 0.42$ \\
$\mathrm{M}$ & -0.56 & -1.64 & -1.00 & 0.68 & -1.39 & -0.25 & $-0.72 \pm 0.42$ \\
\hline & & & & & 0.96 &
\end{tabular}


Table S1 continued

\begin{tabular}{|c|c|c|c|c|c|c|c|}
\hline \multirow{2}{*}{$\frac{\mathrm{A} \cdot \mathrm{A}}{\mathrm{F}}$} & \multicolumn{7}{|c|}{$\Delta \Delta G(\mathrm{kcal} / \mathrm{mol})$} \\
\hline & 0.07 & -1.39 & -1.57 & 0.18 & -1.38 & -0.78 & $-0.99 \pm 0.32$ \\
\hline $\mathrm{P}$ & -0.14 & 2.35 & 0.50 & 0.30 & 2.02 & 0.24 & $1.08 \pm 0.46$ \\
\hline$S$ & 0.57 & 2.01 & 1.45 & 2.15 & 1.40 & 1.66 & $1.73 \pm 0.15$ \\
\hline $\mathrm{T}$ & -0.06 & 0.91 & 1.08 & 1.35 & 0.62 & 1.05 & $1.00 \pm 0.12$ \\
\hline W & 0.54 & -0.93 & -1.60 & 0.70 & -1.17 & 0.27 & $-0.55 \pm 0.44$ \\
\hline Y & 0.15 & -0.27 & -1.30 & 0.48 & -0.22 & 0.57 & $-0.15 \pm 0.34$ \\
\hline V & -0.07 & -1.70 & -0.79 & -0.16 & -1.05 & -0.83 & $-0.91 \pm 0.25$ \\
\hline
\end{tabular}

\begin{tabular}{|c|c|c|c|c|c|c|c|}
\hline & $\Delta \Delta G^{\mathrm{L} 40}$ & $\Delta \Delta G^{\mathrm{P} 116}$ & $\Delta \Delta G^{\mathrm{L} 158}$ & $\Delta \Delta G^{\mathrm{Y} 195}$ & $\Delta \Delta G^{\mathrm{G} 212}$ & $\Delta \Delta G^{\mathrm{V} 261}$ & $\Delta \Delta G_{(2)}$ \\
\hline A & 0 & 0 & 0 & 0 & 0 & 0 & $0 \pm 0$ \\
\hline $\mathrm{R}$ & -0.19 & 2.65 & 1.70 & 1.54 & 2.80 & 1.20 & $1.98 \pm 0.32$ \\
\hline $\mathrm{N}$ & -0.06 & 2.51 & 2.50 & 2.49 & 2.64 & 2.60 & $2.55 \pm 0.03$ \\
\hline D & 0.39 & 3.16 & 2.06 & 2.09 & 3.17 & 3.22 & $2.74 \pm 0.27$ \\
\hline C & 0.48 & 0.17 & 0.25 & -0.06 & 0.42 & -0.12 & $0.13 \pm 0.10$ \\
\hline Q & -0.77 & 0.73 & 0.52 & 1.25 & 1.20 & 0.43 & $0.83 \pm 0.17$ \\
\hline $\mathrm{E}$ & -0.82 & 2.55 & 1.81 & 2.37 & 2.23 & 0.55 & $1.90 \pm 0.36$ \\
\hline G & -0.44 & 0.97 & 0.64 & 1.26 & 1.15 & 0.61 & $0.93 \pm 0.13$ \\
\hline $\mathrm{H}$ & 0.18 & 0.97 & 0.63 & 2.60 & -0.02 & -0.16 & $0.80 \pm 0.49$ \\
\hline I & -0.04 & -1.13 & -1.86 & -1.31 & -1.24 & -1.66 & $-1.44 \pm 0.14$ \\
\hline L & 0.03 & -2.59 & -2.13 & -2.68 & -2.08 & -2.74 & $-2.44 \pm 0.14$ \\
\hline K & 0.72 & 1.06 & 1.36 & 2.65 & 1.88 & 1.57 & $1.70 \pm 0.27$ \\
\hline M & -0.91 & -1.54 & -0.51 & -0.34 & -0.78 & -1.73 & $-0.98 \pm 0.28$ \\
\hline $\mathrm{F}$ & 0.70 & -2.47 & -1.63 & -1.24 & -2.18 & -1.93 & $-1.89 \pm 0.21$ \\
\hline $\mathrm{P}$ & 1.20 & -0.34 & 0.98 & 2.27 & 1.51 & 1.85 & $1.25 \pm 0.45$ \\
\hline S & -0.74 & 1.80 & 1.43 & 1.83 & 2.36 & 1.51 & $1.79 \pm 0.16$ \\
\hline
\end{tabular}


Table S1 continued

\begin{tabular}{|c|c|c|c|c|c|c|c|}
\hline A.A & \multicolumn{7}{|c|}{$\Delta \Delta G(\mathrm{kcal} / \mathrm{mol})$} \\
\hline $\mathrm{T}$ & -0.61 & 1.07 & 0.72 & 1.23 & 1.27 & 0.12 & $0.88 \pm 0.21$ \\
\hline W & 0.18 & -1.41 & -1.12 & -0.23 & -1.08 & -1.25 & $-1.02 \pm 0.21$ \\
\hline Y & 0.27 & 0.41 & 0.00 & 0.22 & -0.65 & -0.76 & $-0.16 \pm 0.23$ \\
\hline $\mathrm{V}$ & 0.10 & -1.90 & -1.53 & -1.67 & -1.22 & -1.08 & $-1.48 \pm 0.15$ \\
\hline
\end{tabular}

\begin{tabular}{|c|c|c|c|c|c|c|c|}
\hline & $\Delta \Delta G^{\mathrm{Q} 94}$ & $\Delta \Delta G^{\mathrm{H} 142}$ & $\Delta \Delta G^{\mathrm{V} 241}$ & $\Delta \Delta G^{\mathrm{F} 69}$ & $\Delta \Delta G^{\mathrm{Y} 177}$ & $\Delta \Delta G^{\mathrm{A} 223}$ & $\Delta \Delta G_{(3)}$ \\
\hline A & 0 & 0 & 0 & 0 & 0 & 0 & $0 \pm 0$ \\
\hline $\mathrm{R}$ & 0.71 & -0.80 & -0.16 & 0.55 & 1.29 & 0.19 & $0.68 \pm 0.32$ \\
\hline $\mathrm{N}$ & -0.23 & -0.32 & 0.92 & 0.14 & 0.46 & 0.50 & $0.37 \pm 0.11$ \\
\hline D & -0.29 & -0.46 & 0.26 & 0.70 & 0.92 & 1.26 & $0.96 \pm 0.16$ \\
\hline C & -0.90 & -0.80 & 0.21 & -1.62 & 0.40 & -1.80 & $-1.01 \pm 0.71$ \\
\hline$Q$ & -0.11 & -0.53 & 1.15 & -0.16 & -0.70 & 0.45 & $-0.14 \pm 0.33$ \\
\hline $\mathrm{E}$ & 0.85 & -0.52 & 0.26 & 0.86 & 0.44 & 1.29 & $0.86 \pm 0.25$ \\
\hline G & 0.02 & -0.39 & -0.05 & 0.63 & 0.67 & 0.44 & $0.58 \pm 0.07$ \\
\hline $\mathrm{H}$ & 0.02 & -1.74 & -0.54 & -0.61 & -0.30 & -0.81 & $-0.57 \pm 0.15$ \\
\hline I & -0.05 & 0.18 & -0.39 & -1.26 & -0.66 & -0.78 & $-0.90 \pm 0.18$ \\
\hline $\mathrm{L}$ & -0.38 & -0.44 & -0.45 & -1.71 & -0.65 & -1.82 & $-1.39 \pm 0.37$ \\
\hline K & 0.68 & -0.14 & 0.11 & 0.28 & 1.28 & -0.09 & $0.49 \pm 0.41$ \\
\hline M & -0.26 & -0.12 & 0.43 & -1.39 & -0.73 & -1.28 & $-1.13 \pm 0.20$ \\
\hline F & -0.34 & -0.44 & -0.71 & -1.61 & -0.81 & -1.71 & $-1.38 \pm 0.28$ \\
\hline P & -0.12 & 0.02 & -0.36 & 0.29 & -0.01 & 0.59 & $0.29 \pm 0.17$ \\
\hline S & -0.15 & -0.18 & 0.36 & 0.61 & 0.38 & 0.75 & $0.58 \pm 0.11$ \\
\hline $\mathrm{T}$ & 0.04 & -0.33 & -0.10 & -0.09 & -0.18 & 0.05 & $-0.07 \pm 0.07$ \\
\hline W & 0.07 & 0.09 & -0.35 & -1.27 & -1.34 & -2.33 & $-1.65 \pm 0.34$ \\
\hline Y & 0.15 & -0.53 & -0.65 & -1.30 & -0.84 & -1.49 & $-1.21 \pm 0.19$ \\
\hline
\end{tabular}


Table S1 continued

\begin{tabular}{|c|c|c|c|c|c|c|c|}
\hline A. A & & & & $\Delta G(\mathrm{kcal} / \mathrm{m}$ & & & \\
\hline V & 0.08 & 0.27 & -0.42 & -1.29 & -0.91 & -0.19 & $-0.80 \pm 0.32$ \\
\hline & $\Delta \Delta G^{\mathrm{Y} 42}$ & $\Delta \Delta G^{\mathrm{N} 156}$ & $\Delta \Delta G^{\mathrm{Y} 114}$ & $\Delta \Delta G^{\mathrm{G} 193}$ & $\Delta \Delta G^{\mathrm{Y} 214}$ & $\Delta \Delta G^{\mathrm{V} 259}$ & $\Delta \Delta G_{(4)}$ \\
\hline A & 0 & 0 & 0 & 0 & 0 & 0 & $0 \pm 0$ \\
\hline$R$ & -0.13 & 1.16 & 0.53 & -0.88 & 0.08 & -0.08 & $-0.09 \pm 0.29$ \\
\hline $\mathrm{N}$ & -0.41 & -1.05 & -0.13 & -0.06 & 0.17 & 0.38 & $0.09 \pm 0.12$ \\
\hline D & -0.35 & 0.28 & 0.62 & -0.21 & 0.37 & 0.40 & $0.30 \pm 0.18$ \\
\hline C & -0.84 & -2.22 & -1.82 & -1.91 & -1.63 & -1.75 & $-1.78 \pm 0.06$ \\
\hline Q & -0.27 & -0.62 & -0.48 & -0.73 & -0.24 & 0.59 & $-0.22 \pm 0.29$ \\
\hline $\mathrm{E}$ & -0.18 & -0.07 & 1.33 & 0.57 & 0.60 & 1.18 & $0.92 \pm 0.20$ \\
\hline G & -0.02 & 0.48 & 0.42 & 0.52 & 0.17 & 0.39 & $0.38 \pm 0.07$ \\
\hline $\mathrm{H}$ & 0.27 & 0.41 & -0.79 & -1.63 & 0.61 & -0.84 & $-0.66 \pm 0.47$ \\
\hline I & -0.08 & -1.89 & -1.13 & -1.46 & -1.11 & -1.06 & $-1.19 \pm 0.09$ \\
\hline L & -0.21 & -1.62 & -1.04 & -1.24 & -1.11 & -1.45 & $-1.21 \pm 0.09$ \\
\hline K & 0.17 & -0.19 & -0.53 & -0.65 & 0.64 & 0.01 & $-0.13 \pm 0.29$ \\
\hline M & -0.18 & -0.31 & -0.96 & -1.07 & -0.99 & -0.97 & $-1.00 \pm 0.03$ \\
\hline $\mathrm{F}$ & -0.04 & -1.94 & -2.03 & -2.07 & -1.32 & -1.57 & $-1.75 \pm 0.18$ \\
\hline $\mathrm{P}$ & 0.20 & 1.37 & -0.75 & 0.35 & -0.14 & 0.10 & $-0.11 \pm 0.24$ \\
\hline S & -0.25 & -0.07 & 0.55 & 0.13 & 0.43 & 0.69 & $0.45 \pm 0.12$ \\
\hline $\mathrm{T}$ & 0.00 & -0.99 & -0.24 & -0.23 & -0.11 & -0.10 & $-0.17 \pm 0.04$ \\
\hline W & 0.16 & -1.97 & -1.88 & -2.53 & -1.27 & -1.86 & $-1.89 \pm 0.25$ \\
\hline Y & 0.40 & -2.11 & -1.47 & -1.57 & -0.78 & -1.50 & $-1.33 \pm 0.18$ \\
\hline V & 0.54 & -1.12 & -0.97 & -1.24 & -0.82 & -0.75 & $-0.95 \pm 0.11$ \\
\hline
\end{tabular}


Table S2. Correlation coefficient $\left(R^{2}\right)$ of transfer free energy scales from different host residues $\left(\Delta \Delta \mathrm{G}^{\text {host }}\right.$ in $\left.\mathrm{kcal} / \mathrm{mol}\right)$ of OmpLA at the same depth-position $i$

\begin{tabular}{|c|c|c|c|c|c|c|}
\hline & $\Delta \Delta G^{F 122}$ & $\Delta \Delta G^{A 164}$ & $\Delta \Delta G^{Y 201}$ & $\Delta \Delta G^{A 206}$ & $\Delta \Delta G^{D 267}$ & \\
\hline$\Delta \Delta G^{F 122}$ & 1.00 & & & & & \\
\hline$\Delta \Delta G^{A 164}$ & 0.88 & 1.00 & & & & \\
\hline$\Delta \Delta G^{Y 201}$ & 0.87 & 0.95 & 1.00 & & & \\
\hline$\Delta \Delta G^{A 206}$ & 0.71 & 0.76 & 0.76 & 1.00 & & \\
\hline$\Delta \Delta G^{D 267}$ & 0.90 & 0.87 & 0.89 & 0.76 & 1.00 & \\
\hline \multicolumn{7}{|c|}{ position $i=3$} \\
\hline & $\Delta \Delta G^{F 75}$ & $\Delta \Delta G^{L 88}$ & $\Delta \Delta G^{V 136}$ & $\Delta \Delta G^{V 171}$ & $\Delta \Delta G^{Y 229}$ & $\Delta \Delta G^{V 235}$ \\
\hline$\Delta \Delta G^{F 75}$ & 1.00 & & & & & \\
\hline$\Delta \Delta G^{F 75}$ & 1.00 & & & & & \\
\hline$\Delta \Delta G^{V 136}$ & 0.43 & 0.53 & 1.00 & & & \\
\hline$\Delta \Delta G^{V 171}$ & 0.39 & 0.48 & 0.65 & 1.00 & & \\
\hline$\Delta \Delta G^{Y 229}$ & 0.85 & 0.78 & 0.59 & 0.57 & 1.00 & \\
\hline$\Delta \Delta G^{V 235}$ & 0.11 & 0.23 & 0.23 & 0.51 & 0.14 & 1.00 \\
\hline \multicolumn{7}{|c|}{ position $i=2$} \\
\hline & $\Delta \Delta G^{D 36}$ & $\Delta \Delta G^{L 120}$ & $\Delta \Delta G^{L 162}$ & $\Delta \Delta G^{I 199}$ & $\Delta \Delta G^{L 208}$ & $\Delta \Delta G^{L 265}$ \\
\hline$\Delta \Delta G^{D 36}$ & 1.00 & & & & & \\
\hline$\Delta \Delta G^{L 120}$ & 0.08 & 1.00 & & & & \\
\hline$\Delta \Delta G^{L 162}$ & 0.01 & 0.79 & 1.00 & & & \\
\hline$\Delta \Delta G^{I 199}$ & 0.04 & 0.89 & 0.95 & 1.00 & & \\
\hline$\Delta \Delta G^{L 208}$ & 0.03 & 0.83 & 0.84 & 0.86 & 1.00 & \\
\hline$\Delta \Delta G^{L 265}$ & 0.01 & 0.55 & 0.64 & 0.64 & 0.71 & 1.00 \\
\hline
\end{tabular}


Table s2 continued

\begin{tabular}{|c|c|c|c|c|c|c|}
\hline \multicolumn{7}{|c|}{ position $i=1$} \\
\hline & $\Delta \Delta G^{L 73}$ & $\Delta \Delta G^{A 90}$ & $\Delta \Delta G^{M 138}$ & $\Delta \Delta G^{V 173}$ & $\Delta \Delta G^{L 227}$ & $\Delta \Delta G^{L 237}$ \\
\hline$\Delta \Delta G^{L 73}$ & 1.00 & & & & & \\
\hline$\Delta \Delta G^{A 90}$ & 0.90 & 1.00 & & & & \\
\hline$\Delta \Delta G^{M 138}$ & 0.53 & 0.48 & 1.00 & & & \\
\hline$\Delta \Delta G^{V 173}$ & 0.82 & 0.71 & 0.75 & 1.00 & & \\
\hline$\Delta \Delta G^{L 227}$ & 0.87 & 0.87 & 0.47 & 0.65 & 1.00 & \\
\hline$\Delta \Delta G^{L 237}$ & 0.44 & 0.37 & 0.58 & 0.63 & 0.38 & 1.00 \\
\hline
\end{tabular}

\begin{tabular}{|c|c|c|c|c|c|c|}
\hline & $\Delta \Delta G^{N 38}$ & $\Delta \Delta G^{L 118}$ & $\Delta \Delta G^{T 160}$ & $\Delta \Delta G^{L 197}$ & $\Delta \Delta G^{A 210}$ & $\Delta \Delta G^{V 263}$ \\
\hline$\Delta \Delta G^{N 38}$ & 1.00 & & & & & \\
\hline$\Delta \Delta G^{L 118}$ & 0.14 & 1.00 & & & & \\
\hline$\Delta \Delta G^{T 160}$ & 0.13 & 0.86 & 1.00 & & & \\
\hline$\Delta \Delta G^{L 197}$ & 0.04 & 0.89 & 0.82 & 1.00 & & \\
\hline$\Delta \Delta G^{A 210}$ & 0.18 & 0.95 & 0.88 & 0.84 & 1.00 & \\
\hline$\Delta \Delta G^{V 263}$ & 0.19 & 0.92 & 0.72 & 0.76 & 0.88 & 1.00 \\
\hline
\end{tabular}

\begin{tabular}{|c|c|c|c|c|c|c|}
\hline & $\Delta \Delta G^{L 71}$ & $\Delta \Delta G^{Y 92}$ & $\Delta \Delta G^{Y 140}$ & $\Delta \Delta G^{P 175}$ & $\Delta \Delta G^{L 225}$ & $\Delta \Delta G^{T 239}$ \\
\hline$\Delta \Delta G^{L 71}$ & 1.00 & & & & & \\
\hline$\Delta \Delta G^{Y 92}$ & 0.81 & 1.00 & & & & \\
\hline$\Delta \Delta G^{Y 140}$ & 0.70 & 0.67 & 1.00 & & & \\
\hline$\Delta \Delta G^{P 175}$ & 0.42 & 0.35 & 0.66 & 1.00 & & \\
\hline$\Delta \Delta G^{L 225}$ & 0.94 & 0.76 & 0.59 & 0.34 & 1.00 & \\
\hline$\Delta \Delta G^{T 239}$ & 0.79 & 0.65 & 0.86 & 0.64 & 0.64 & 1.00 \\
\hline
\end{tabular}


Table s2 continued

\begin{tabular}{|c|c|c|c|c|c|c|}
\hline \multicolumn{7}{|c|}{ position $i=-2$} \\
\hline & $\Delta \Delta G^{L 40}$ & $\Delta \Delta G^{P 116}$ & $\Delta \Delta G^{L 158}$ & $\Delta \Delta G^{Y 195}$ & $\Delta \Delta G^{G 212}$ & $\Delta \Delta G^{V 261}$ \\
\hline$\Delta \Delta G^{L 40}$ & 1.00 & & & & & \\
\hline$\Delta \Delta G^{P 116}$ & 0.05 & 1.00 & & & & \\
\hline$\Delta \Delta G^{L 158}$ & 0.02 & 0.89 & 1.00 & & & \\
\hline$\Delta \Delta G^{Y 195}$ & 0.00 & 0.72 & 0.86 & 1.00 & & \\
\hline$\Delta \Delta G^{G 212}$ & 0.03 & 0.87 & 0.92 & 0.75 & 1.00 & \\
\hline$\Delta \Delta G^{V 261}$ & 0.01 & 0.74 & 0.85 & 0.74 & 0.88 & 1.00 \\
\hline \multicolumn{7}{|c|}{ position $i=-3$} \\
\hline & $\Delta \Delta G^{F 69}$ & $\Delta \Delta G^{Q 94}$ & $\Delta \Delta G^{H 142}$ & $\Delta \Delta G^{Y 177}$ & $\Delta \Delta G^{A 223}$ & $\Delta \Delta G^{V 241}$ \\
\hline$\Delta \Delta G^{F 69}$ & 1.00 & & & & & \\
\hline$\Delta \Delta G^{Q 94}$ & 0.26 & 1.00 & & & & \\
\hline$\Delta \Delta G^{H 142}$ & 0.00 & 0.00 & 1.00 & & & \\
\hline$\Delta \Delta G^{Y 177}$ & 0.57 & 0.10 & 0.05 & 1.00 & & \\
\hline$\Delta \Delta G^{A 223}$ & 0.83 & 0.15 & 0.00 & 0.39 & 1.00 & \\
\hline$\Delta \Delta G^{V 241}$ & 0.19 & 0.01 & 0.00 & 0.11 & 0.25 & 1.00 \\
\hline \multicolumn{7}{|c|}{ position $i=-4$} \\
\hline & $\Delta \Delta G^{Y 42}$ & $\Delta \Delta G^{Y 114}$ & $\Delta \Delta G^{N 156}$ & $\Delta \Delta G^{G 193}$ & $\Delta \Delta G^{Y 214}$ & $\Delta \Delta G^{V 259}$ \\
\hline$\Delta \Delta G^{Y 42}$ & 1.00 & & & & & \\
\hline$\Delta \Delta G^{Y 114}$ & 0.03 & 1.00 & & & & \\
\hline$\Delta \Delta G^{N 156}$ & 0.01 & 0.49 & 1.00 & & & \\
\hline$\Delta \Delta G^{G 193}$ & 0.01 & 0.72 & 0.46 & 1.00 & & \\
\hline$\Delta \Delta G^{Y 214}$ & 0.01 & 0.68 & 0.57 & 0.54 & 1.00 & \\
\hline$\Delta \Delta G^{V 259}$ & 0.02 & 0.82 & 0.48 & 0.82 & 0.71 & 1.00 \\
\hline
\end{tabular}




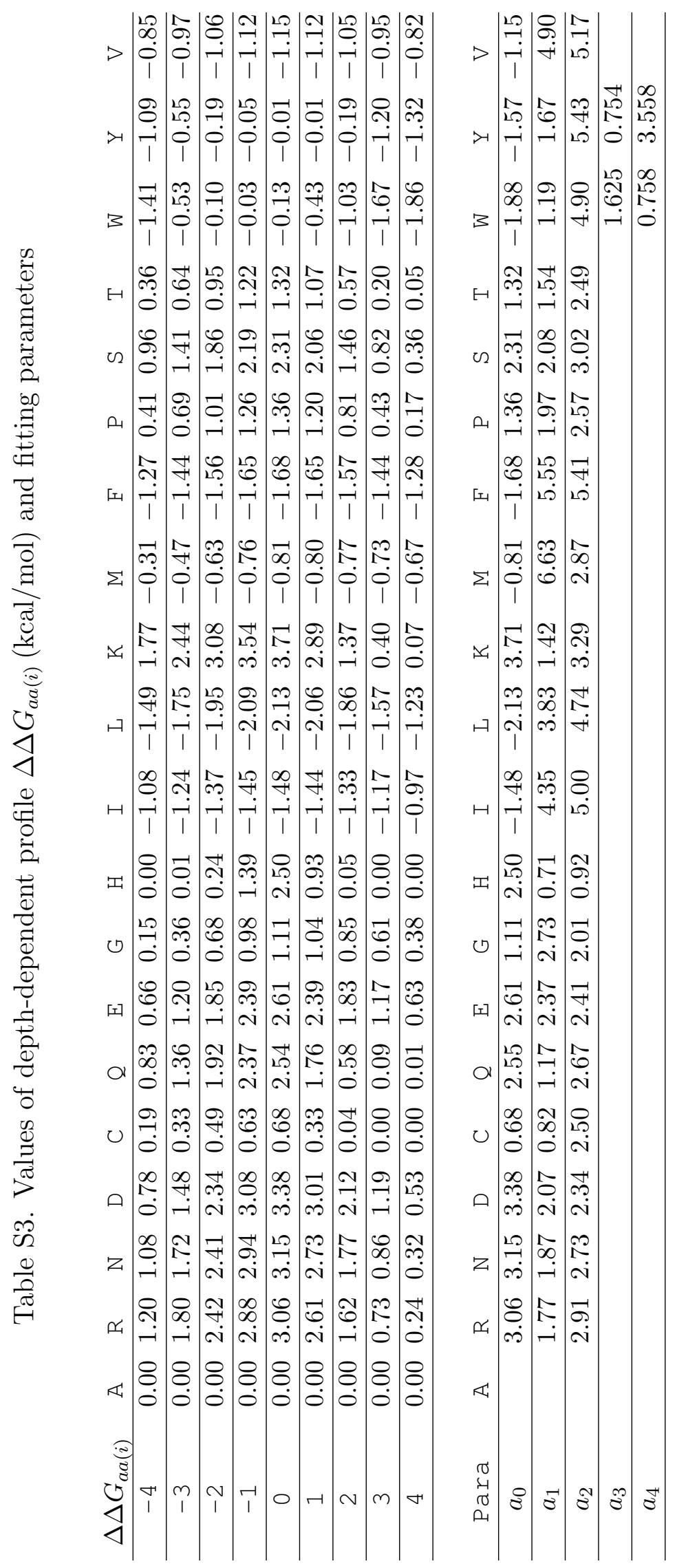


Table S4. Differences in the folding free energy of the TM regions of OMPs in the NCIN and the NC-OUT topology calculated using the additive model by summing up the transfer free energy of all lipid facing TM residues $\left(\Delta \Delta \mathrm{G}_{\text {topo }}^{\text {Addive }}\right.$ in $\left.\mathrm{kcal} / \mathrm{mol}\right)$ and by us-

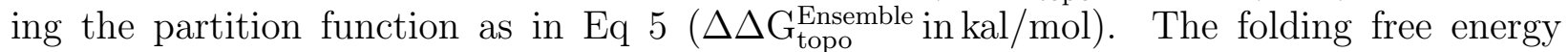
$\left(\Delta \mathrm{G}_{\mathrm{NC}-\mathrm{IN}}^{\text {Ensemble }}\right.$ in $\left.\mathrm{kcal} / \mathrm{mol}\right)$ calculated using Eq. 5 is also listed.

\begin{tabular}{|c|c|c|c|c|}
\hline Protein & $\mathrm{PDB}$ ID & $\Delta \Delta \mathrm{G}_{\text {topo }}^{\text {Additive }}$ & $\Delta \Delta \mathrm{G}_{\text {topo }}^{\text {Ensemble }}$ & $\Delta \mathrm{G}_{\mathrm{NC}-\mathrm{IN}}^{\text {Ensemble }}$ \\
\hline OmpA & $1 B X W$ & -2.20 & 10.76 & -85.80 \\
\hline OmpX & 1QJ8 & 2.38 & 9.57 & -80.80 \\
\hline NspA & $1 \mathrm{P} 4 \mathrm{~T}$ & 0.20 & 3.33 & -85.03 \\
\hline OmpW & $2 \mathrm{~F} 1 \mathrm{~T}$ & -2.34 & 1.21 & -79.58 \\
\hline PagP & $1 \mathrm{THQ}$ & 3.71 & 1.81 & -78.90 \\
\hline OpcA & $1 \mathrm{~K} 24$ & 3.03 & 12.80 & -101.65 \\
\hline Ompt & 1178 & 0.32 & 8.95 & -97.57 \\
\hline OmpLA & 1QD 6 & 2.09 & 11.51 & -115.17 \\
\hline $\mathrm{NalP}$ & 1UYN & 3.27 & 13.37 & -119.08 \\
\hline FadL & $1 \mathrm{~T} 16$ & -1.30 & 12.98 & -140.39 \\
\hline OmpG & $2 \mathrm{~F} 1 \mathrm{C}$ & -0.59 & 4.81 & -144.21 \\
\hline FepA & $1 \mathrm{FEP}$ & 1.65 & 26.98 & -201.88 \\
\hline FhuA & $2 \mathrm{FCP}$ & -0.85 & 22.34 & -205.09 \\
\hline FecA & $1 \mathrm{KMO}$ & 1.65 & 16.22 & -206.17 \\
\hline BtuB & $1 \mathrm{NQE}$ & -0.92 & 19.35 & -189.49 \\
\hline FptA & $1 \mathrm{XKW}$ & 0.08 & 15.54 & -203.87 \\
\hline Porin & $1 \mathrm{PRN}$ & 2.40 & 9.06 & -138.77 \\
\hline Porin & $2 \mathrm{POR}$ & 3.95 & 7.03 & -150.68 \\
\hline OmpF & $2 \mathrm{OMF}$ & 5.07 & 11.13 & -141.27 \\
\hline Omp 32 & $1 \mathrm{E} 54$ & 2.11 & 16.13 & -146.27 \\
\hline PorinP & $204 \mathrm{~V}$ & 2.73 & 18.44 & -153.98 \\
\hline LamB & $2 \mathrm{MPR}$ & 4.66 & 7.60 & -156.50 \\
\hline $\operatorname{Scr} Y$ & $1 \mathrm{AOS}$ & 7.95 & 16.48 & -157.27 \\
\hline BamA & $4 \mathrm{~K} 3 \mathrm{~B}$ & -2.17 & 8.32 & -153.36 \\
\hline
\end{tabular}

There is correlation between the folding free energy and the number of strands in the OMPs. However, OMPs of the same family can have different folding energy. Among the five OMPs belonging to the TonB-dependent transporter family, BtuB (PDB id: $1 \mathrm{NQE}$ ) is less stable than the other four proteins (PDB id: 1FEP, 1KMO, 1NQE, and $1 \mathrm{XKW})$. The folding free energy of BtuB is $7.2 \%$ lower than the average folding the other four members. 


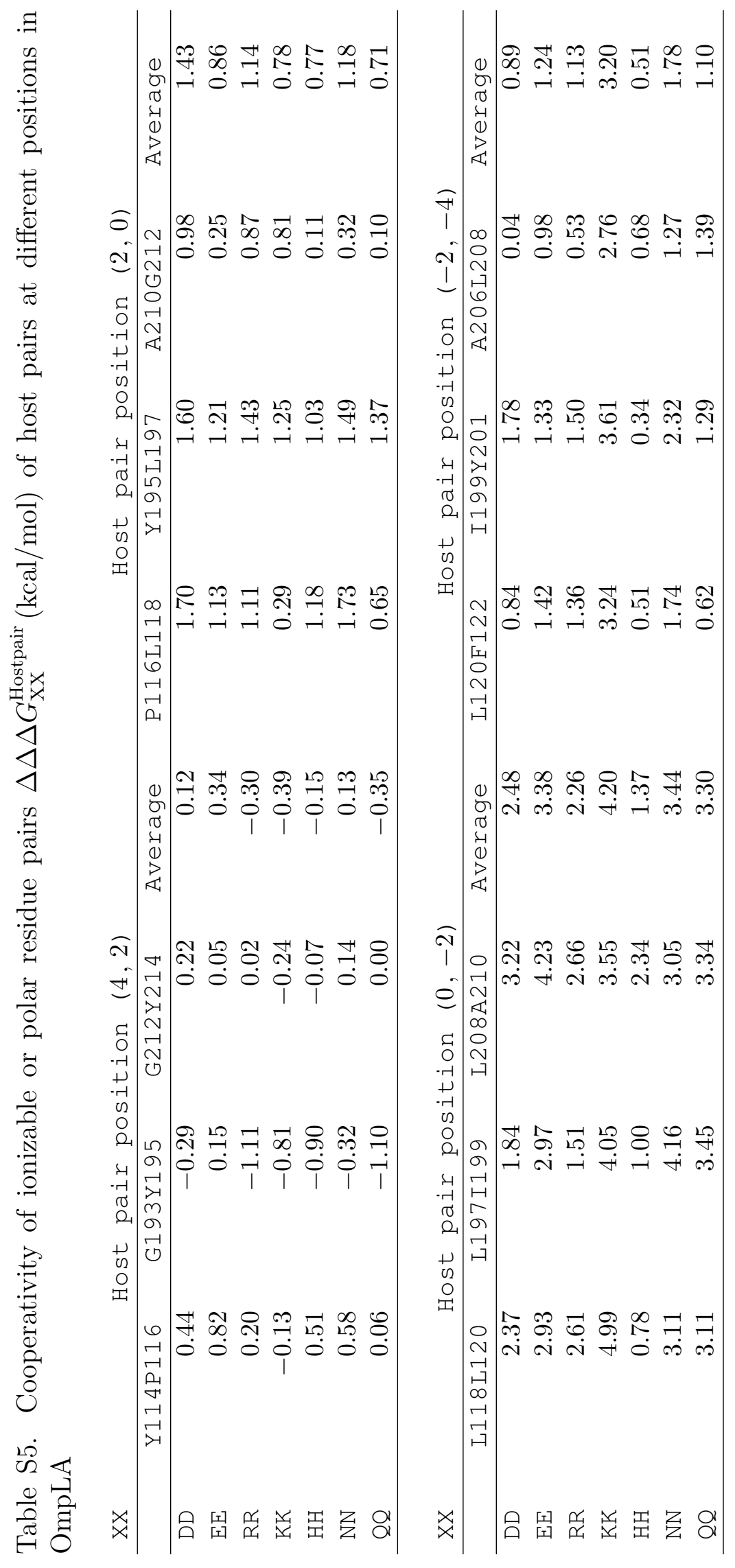


Table S6. Values of experimental and knowledge-based amino acid hydrophobicity scales in $\mathrm{kcal} / \mathrm{mol}$.

\begin{tabular}{|c|c|c|c|c|c|c|}
\hline & \multicolumn{3}{|c|}{ experimental } & \multicolumn{3}{|c|}{ knowledge-based } \\
\hline A.A & $\Delta \Delta \mathrm{G}_{\mathrm{w}, \mathrm{l}}^{\mathrm{O}}$ & $\Delta \Delta \mathrm{G}_{\mathrm{app}}^{\mathrm{aa}}$ & $\Delta \Delta \mathrm{G}_{\text {octanol }}$ & $E_{z} \beta$ & Slusky-Dunbrack & $E_{b}$ \\
\hline A & 0 & 0.11 & 0.50 & -0.8 & 0.13 & -0.69 \\
\hline $\mathrm{R}$ & 3.71 & 2.58 & 1.81 & 1.4 & -1.06 & 2.66 \\
\hline $\mathrm{N}$ & 3.47 & 2.05 & 0.85 & 0.7 & -0.69 & 2.30 \\
\hline $\mathrm{D}$ & 2.95 & 3.49 & 3.64 & 1.3 & -0.63 & 2.66 \\
\hline C & 0.49 & -0.13 & -0.02 & $N A$ & $N A$ & 4.61 \\
\hline Q & 3.01 & 2.36 & 0.77 & 0.7 & -0.69 & 2.21 \\
\hline $\mathrm{E}$ & 1.64 & 2.68 & 3.63 & 1.1 & -2.48 & 4.61 \\
\hline G & 1.72 & 0.74 & 1.15 & 0 & 0.04 & 0.27 \\
\hline $\mathrm{H}$ & 4.76 & 2.06 & 0.11 & 1.2 & -0.25 & 1.90 \\
\hline I & -1.56 & -0.60 & -1.12 & -1.0 & 0.10 & -0.83 \\
\hline L & -1.81 & -0.55 & -1.25 & -2.0 & 0.35 & -0.91 \\
\hline K & 5.39 & 2.71 & 2.80 & 1.3 & -0.98 & 3.51 \\
\hline M & -0.76 & -0.10 & -0.67 & $N A$ & 0 & -0.46 \\
\hline $\mathrm{F}$ & -2.20 & -0.32 & -1.71 & -1.9 & 0.21 & -0.25 \\
\hline $\mathrm{P}$ & -1.52 & 2.23 & 0.14 & 0.8 & 0.31 & 0.05 \\
\hline S & 1.83 & 0.84 & 0.46 & 0.9 & -0.11 & 1.31 \\
\hline $\mathrm{T}$ & 1.78 & 0.52 & 0.25 & $N A$ & -0.30 & 0.40 \\
\hline W & -0.38 & 0.30 & -2.09 & -0.4 & -0.44 & 0.26 \\
\hline$Y$ & -1.09 & 0.68 & -0.71 & -0.0 & -0.21 & 0.17 \\
\hline V & -0.78 & -0.31 & -0.46 & -1.5 & 0.05 & -0.96 \\
\hline
\end{tabular}

$\Delta \Delta \mathrm{G}_{\mathrm{w}, \mathrm{l}}^{\mathrm{o}}$ : Moon and Fleming's whole-protein scale ${ }^{16}$

$\Delta \Delta \mathrm{G}_{\mathrm{app}}^{\mathrm{aa}}$ : Hessa et al's biological scale ${ }^{21}$

$\Delta \Delta \mathrm{G}_{\text {octanol }}$ : Wimley and White's octanol scale ${ }^{22}$

$E_{z} \beta$ : Hsieh et al's $E_{z} \beta$ scale ${ }^{19}$

Slusky-Dunbrack: Slusky and Dunbrack's scale 20

$E_{b}$ : Jackups and Liang's scale ${ }^{4}$ 


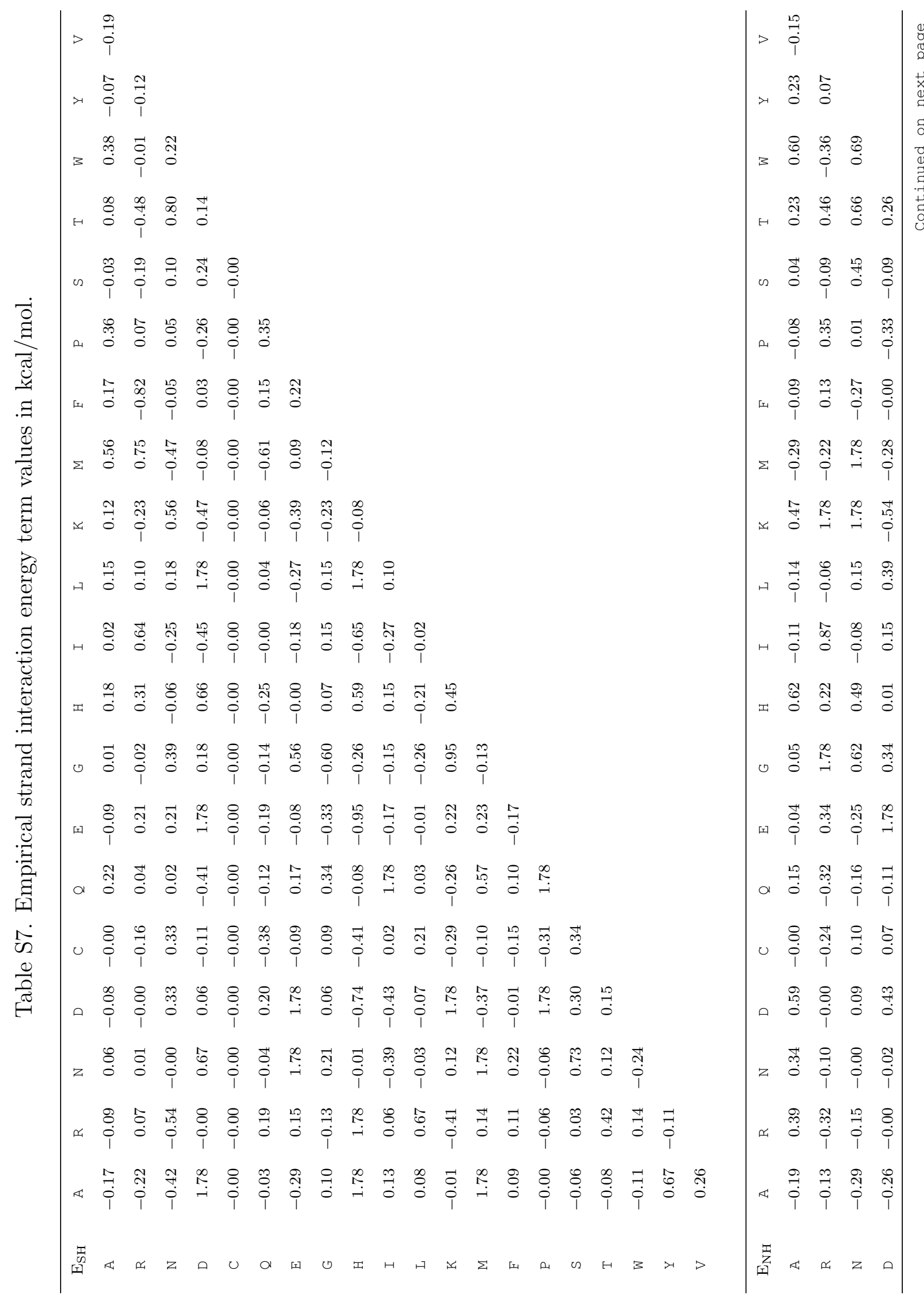




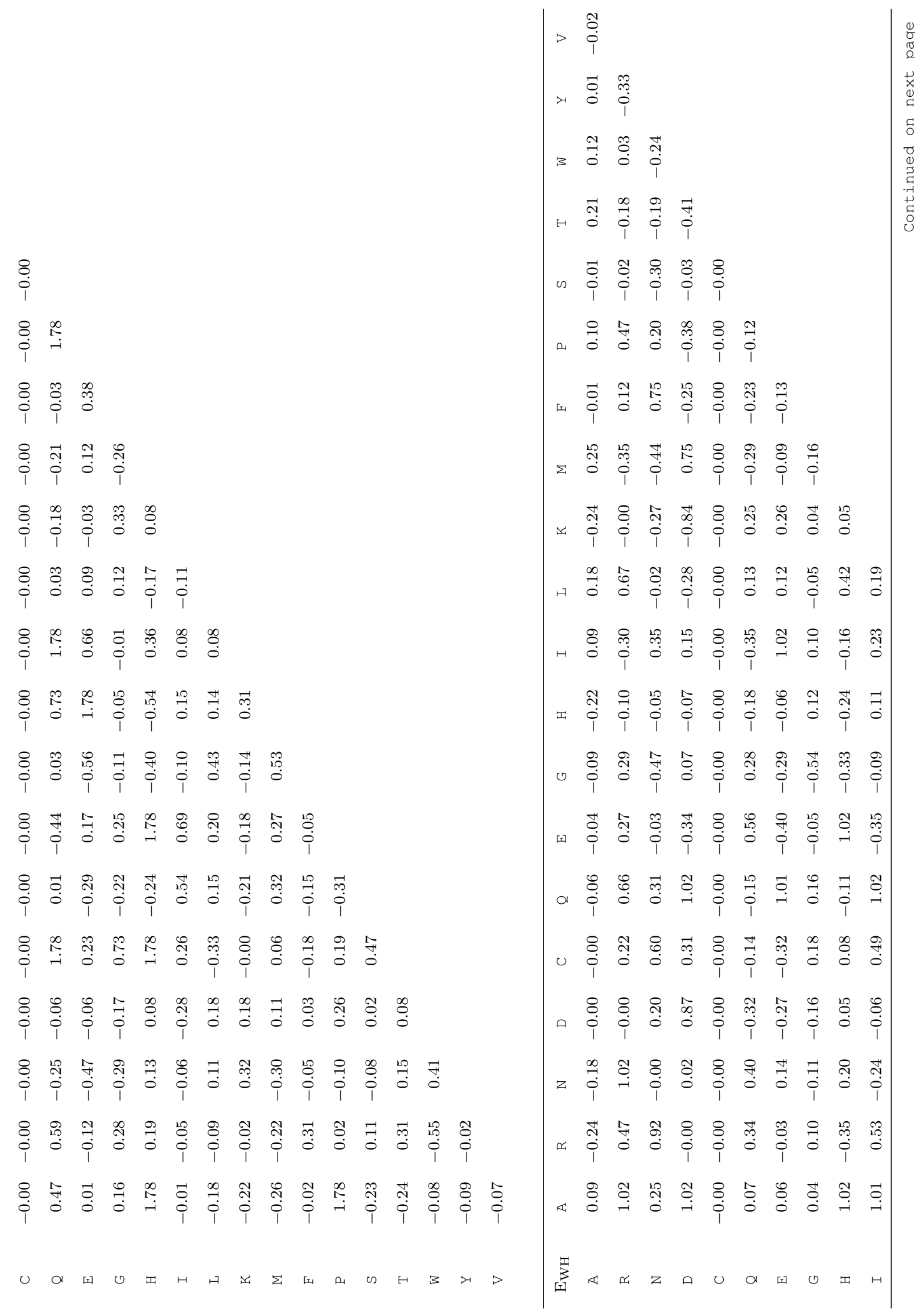




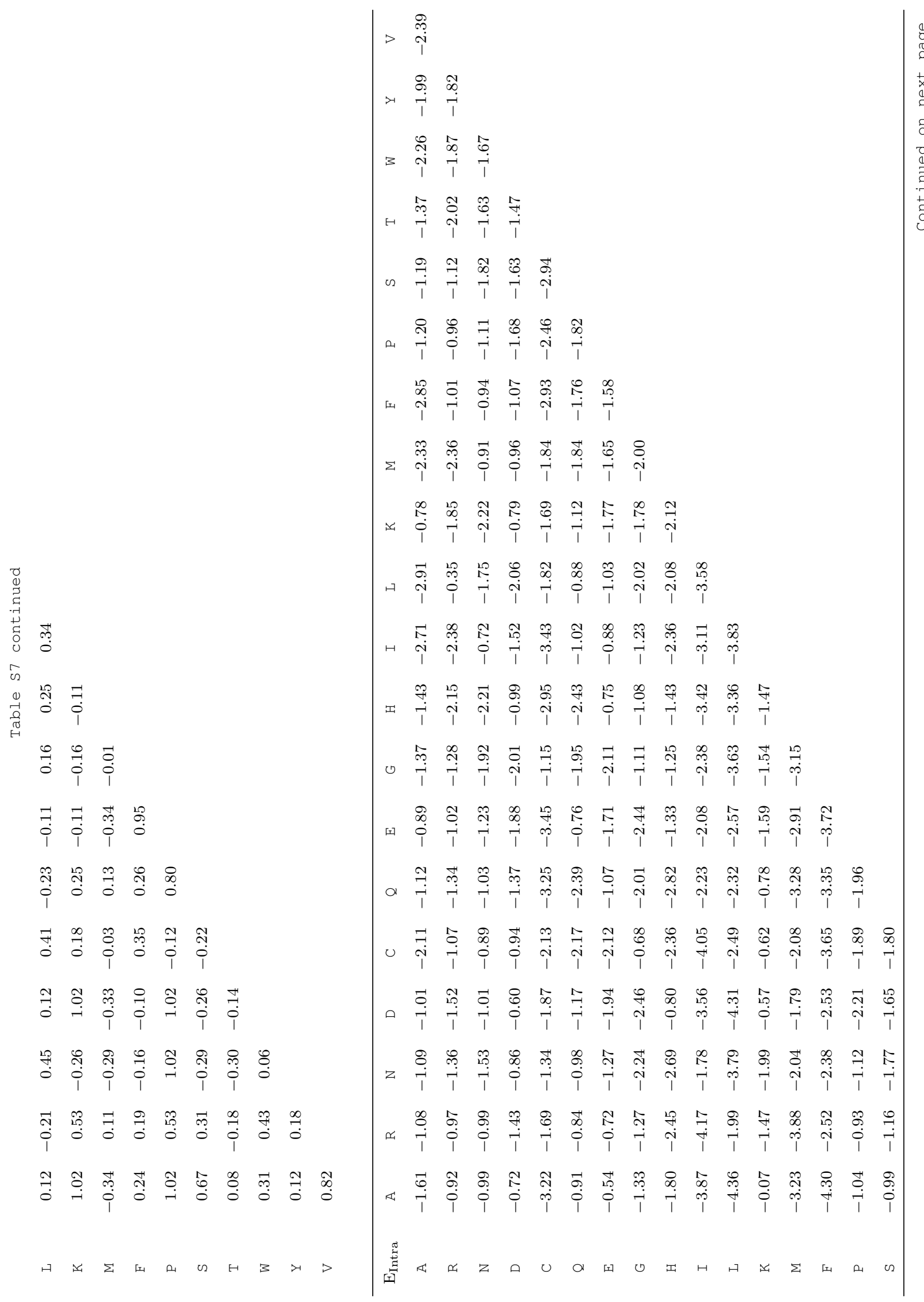




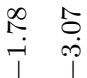

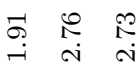

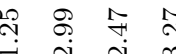

$\uparrow$ i

H $3>>$ 
Table S8. Single burial energy term values in $\mathrm{kcal} / \mathrm{mol}$.

\begin{tabular}{lrrrrrrrr} 
AA & E-CAP & E-HG-IN & E-HG-OUT & C-IN & C-OUT & P-HG-IN & P-HG-OUT & P-CAP \\
\hline A & 0.20 & 0.19 & 0.28 & 0.01 & -0.41 & 0.19 & 0.39 & 0.03 \\
R & 0.12 & -0.24 & 0.83 & -0.30 & 1.57 & -0.15 & 0.06 & -0.23 \\
N & -0.23 & -0.14 & 0.59 & -0.09 & 1.36 & -0.01 & 0.66 & -0.24 \\
D & -0.34 & -0.15 & 1.90 & 0.25 & 1.57 & 0.02 & 0.23 & -0.30 \\
$\mathrm{C}$ & 0.83 & 0.83 & 0.83 & 0.83 & 0.83 & 0.83 & 0.83 & -0.88 \\
$\mathrm{Q}$ & 0.25 & -0.10 & 0.21 & -0.21 & 1.31 & -0.47 & -0.11 & -0.10 \\
$\mathrm{E}$ & -0.16 & -0.26 & 0.83 & -0.32 & 0.83 & -0.26 & 0.41 & -0.08 \\
$\mathrm{G}$ & -0.06 & 0.20 & 0.25 & -0.33 & 0.16 & -0.06 & 0.64 & 0.02 \\
$\mathrm{H}$ & -0.09 & -0.18 & -0.46 & 0.20 & 1.12 & 0.64 & -0.54 & 0.06 \\
$\mathrm{I}$ & 0.13 & 0.50 & -0.32 & 0.60 & -0.49 & 0.09 & 0.01 & 0.25 \\
$\mathrm{~L}$ & 0.08 & 0.46 & -0.34 & 0.54 & -0.54 & 0.25 & 0.12 & 0.40 \\
$\mathrm{~K}$ & 0.03 & -0.51 & 1.21 & -0.03 & 2.08 & 0.22 & -0.19 & -0.26 \\
$\mathrm{M}$ & 0.15 & 0.49 & 0.35 & 0.02 & -0.27 & -0.29 & -0.05 & 0.07 \\
$\mathrm{~F}$ & -0.17 & 0.98 & -0.57 & 0.54 & -0.15 & 0.73 & -0.36 & 0.26 \\
$\mathrm{P}$ & -0.57 & 0.15 & 0.77 & 1.43 & 0.03 & 0.83 & 0.20 & -0.10 \\
$\mathrm{~S}$ & 0.13 & -0.38 & 0.57 & -0.22 & 0.77 & -0.19 & 0.43 & -0.16 \\
$\mathrm{~T}$ & -0.01 & -0.35 & 0.25 & -0.06 & 0.24 & -0.27 & 0.19 & 0.06 \\
$\mathrm{~W}$ & 0.20 & 0.60 & -0.67 & 0.40 & 0.15 & 0.46 & -0.72 & 0.25 \\
$\mathrm{Y}$ & 0.82 & 0.33 & -0.54 & 0.06 & -0.10 & 0.16 & -0.64 & 0.30 \\
$\mathrm{~V}$ & 0.28 & 0.53 & -0.32 & 0.64 & -0.57 & 0.03 & 0.02 & 0.35 \\
\hline
\end{tabular}

